\title{
A combined path-percolation - Lattice-Boltzmann model applied to multiphase mass transfer in porous media
}

\author{
Ozgur Cekmer ${ }^{\mathrm{a}}$, Sukkee Um ${ }^{\mathrm{b}}$, Matthew M. Mench ${ }^{\mathrm{a}, \mathrm{c}, 1}$
}

${ }^{a}$ Electrochemical Energy Storage and Conversion Laboratory, Department of Mechanical, Aerospace, and Biomedical Engineering, The University of Tennessee, Knoxville, TN 37996, USA

${ }^{\mathrm{b}}$ School of Mechanical Engineering, Hanyang University, Seoul 133-791

${ }^{c}$ Energy and Transportation Science Division, Oak Ridge National Laboratory, Oak Ridge, TN 37831, USA

Submitted to International Journal of

Heat and Mass Transfer

on

05.08.2015

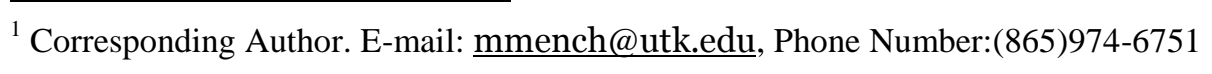




\begin{abstract}
In this work, single-component single-phase, and single-component multi-phase LatticeBoltzmann models were developed to investigate the effects of liquid formation on mass transfer in porous channels via path-percolation theory. A two-dimensional lattice with nine velocity components was used in both lattice-Boltzmann models. A confidence level of $99 \%$ was utilized to obtain statistical results of porosity, effective porosity, and tortuosity of the system with $0 \%$, $10 \%$, and $20 \%$ liquid formation. Velocity distributions in randomly generated inhomogeneous porous channels with different solid-liquid-vapor combinations were analyzed. The statistical results show that the porosity range of the initially generated porous media lies between the specified error limit of 0.001 determined by the confidence level study for all three cases with $70 \%, 80 \%$, and $90 \%$ target porosity. When target porosity decreases, the difference between porosity and effective porosity increases and the effective porosity range gets wider than the range of porosity. Effective diffusion coefficient decreases with increase in liquid formation, since the effective porosity decreases. An application programming interface called OpenMP was implemented on the developed serial in-house program and the effects of 1 to 4 threads on program performance and efficiency were investigated. The maximum speedup and performance gained are 3.3553 and 1.275 GFlops for 4 threads of a personal computer with a 38.4 GFlops peak performance.
\end{abstract}

\title{
Keywords
}

Lattice-Boltzmann Model; Path-Percolation Theory; Porous Media; Multiphase Flow; OpenMP; Statistical

\section{Nomenclature}

a

$b$

$C L$

c

$c_{s}$

$D_{\text {bulk }}$

$D_{\text {eff }}$

F

$f$

$f^{e q}$

A parameter to include intermolecular attractive force into the van der Waals equation of state, $\left(\mathrm{m}^{6} \mathrm{~Pa} / \mathrm{mol}^{2}\right)$

A parameter to include the effects of non-negligible molecule volume into the van der Waals equation of state, $\left(\mathrm{m}^{3} / \mathrm{mol}\right)$

Confidence level

Unit lattice velocity, $(\mathrm{m} / \mathrm{s})$

Unit lattice sound velocity, $(\mathrm{m} / \mathrm{s})$

Bulk diffusion coefficient, $\left(\mathrm{m}^{2} / \mathrm{s}\right)$

Effective diffusion coefficient, $\left(\mathrm{m}^{2} / \mathrm{s}\right)$

Intermolecular force, $(N)$

The probability distribution function

Equivalent distribution function 


\begin{tabular}{|c|c|}
\hline G & Intermolecular interaction strength \\
\hline$k$ & Lattice index \\
\hline$N$ & Number of floating point operations in the code \\
\hline$N_{R A E}$ & $\begin{array}{l}\text { Number of the representative area elements in a statistical representative area } \\
\text { element set }\end{array}$ \\
\hline$n$ & Total history number \\
\hline$n_{c}$ & Number of threads used in parallel code \\
\hline$n_{m}$ & Number of moles of the fluid \\
\hline$P$ & Pressure of the fluid, $(\mathrm{Pa})$ \\
\hline$P_{\text {peak }}$ & Peak performance of the computer, $(F L O P / s)$ \\
\hline $\operatorname{Pr}$ & Probability of any event to occur \\
\hline$p$ & The probability of a pore to occur in a node \\
\hline$q$ & The probability of a solid to occur in a node \\
\hline$R$ & Gas constant of the fluid, $(\mathrm{J} / \mathrm{mol} \mathrm{K})$ \\
\hline$S$ & Speedup \\
\hline$T$ & Temperature of the fluid, $(K)$ \\
\hline$T_{1}$ & Runtime of the code with a single thread, $(s)$ \\
\hline$T_{n_{e}}$ & Runtime of the code with $n_{c}$ threads, $(s)$ \\
\hline$t$ & Time, $(s)$ \\
\hline$u$ & Velocity, $u=\sqrt{u_{x}^{2}+u_{y}^{2}},(\mathrm{~m} / \mathrm{s})$ \\
\hline$u_{x}$ & Velocity in horizontal direction, $(\mathrm{m} / \mathrm{s})$ \\
\hline$u_{y}$ & Velocity in vertical direction, $(\mathrm{m} / \mathrm{s})$ \\
\hline$V$ & Volume of the fluid, $\left(\mathrm{m}^{3}\right)$ \\
\hline$w$ & Weighting factor of the lattice components \\
\hline$\hat{x}$ & Arithmetic average of any property \\
\hline$x$ & Horizontal distance, $(m)$ \\
\hline$y$ & Verical distance, $(m)$ \\
\hline
\end{tabular}




\section{Greek Letters}

$\begin{array}{ll}\varepsilon & \text { Error between the true and estimated probabilities of an event } \\ \varepsilon_{\text {parallel }} & \text { Efficiency of the parallel code } \\ \rho & \text { Density of the fluid, }\left(\mathrm{kg} / \mathrm{m}^{3}\right) \\ \rho_{0} & \text { An arbitrary parameter, }\left(\mathrm{kg} / \mathrm{m}^{3}\right) \\ \tau & \text { Tortuosity } \\ \tau_{\text {eff }} & \text { Effective tortuosity } \\ \phi & \text { Porosity } \\ \phi_{\text {eff }} & \text { Effective porosity } \\ \psi & \text { Intermolecular interaction potential } \\ \psi_{0} & \text { An arbitrary parameter }\end{array}$

\section{Double-struck Letters}

$\mathbb{G} \quad$ Gaussian function

\section{Introduction}

The Lattice-Boltzmann equation was derived by Ludwig Boltzmann in 1872, and is counted as the cornerstone of the kinetic theory of gases [1]. The Lattice-Boltzmann Method (LBM) is an explicit technique based on Ludwig Boltzmann's kinetic theory of gases [2] used to simulate transport equations. In kinetic theory, a statistical treatment is performed for fluid particles, and the actions of these particles are described by streaming and collision mechanisms. Detailed information about the kinetic theory of gases can be found in references [1-4]. The LBM treats a group of particles as a lattice unit and investigates the behavior of the units in the system. In terms of computational fluid dynamics, LBM can be used to simulate single and multiphase flows, and in this study it was applied to simulate two-phase momentum transfer in porous media. Many studies with multi-phase LBM in porous media can be found in literature [5-13]. Among these, the authors decided to develop a single-component multiphase (SCMP) LBM model similar to that in the study of Chau et al. [14]. They used a two-dimensional, SCMP LBM code to investigate the effects of gravity on liquid formation, hence the gaseous diffusion in partially saturated porous media. They found that the relative (effective) diffusion coefficient 
shows about a $25 \%$ difference between different liquid configurations under zero gravity. The target of the current study is observing the effects of liquid formation on diffusivity by determining the effective porosity and tortuosity in randomly generated inhomogeneous porous channels with $0 \%, 10 \%$, and $20 \%$ liquid formation based on a statistical point of view.

A statistical based "path-percolation theory" was developed by Jung et al. [15] to investigate the electrical property variations in inhomogeneous porous media. A process called "cluster labelling" was applied for a path determination scheme. Cekmer et al. [16] applied this theory with a combination of a single-phase lattice-Boltzmann model to investigate the mass transfer in randomly generated inhomogeneous porous media. A new effective diffusion model was developed in terms of effective porosity obtained by eliminating the orphan pores, which were not connected to the pathways between the inlet and outlet of a channel. This model was also tested and compared with two well-known diffusion models $[17,18]$. In this study, the pathpercolation theory was utilized to generate random inhomogeneous porous channels and determine the effective porosity after liquid particles plugged some of the paths for the particles in vapor-phase.

In addition, an application programming interface called open multi-processing (OpenMP) was implemented to the code to gain higher performance and speedup, which is defined as the runtime ratio of the codes with single- and multi- threads. OpenMP was applied to solve SCMP LBM for many representative area elements of random porous channels by multi threads simultaneously instead of sharing a single LBM simulation to avoid the time lost by message passing between the nodes. However, the main novelty of this article is its statistical results of multi-phase effective diffusion process for a number of different solid-vapor-liquid configurations determined by the confidence level studies.

The motivation of this work is to develop a statistical based diffusion behavior estimation of two-phase systems in inhomogeneous porous media, following on previously developed work valid for single-phase flow only [16]. This work will provide a better understanding of the effects of internal structure of the porous channels on multi-phase mass flow than macroscopic approaches. The novel methods introduced and the outcome of the work can be utilized in any application area of heterogeneous porous media involving single- and multi-phase mass and momentum transport. Furthermore, the increased performance of the computational model with OpenMP implementation is another significant improvement, since it has a potential to provide a more accurate statistical results with a higher confidence level using high performance computing systems.

\section{Methodology}

Three different models were used in this study to investigate the effects of liquid formation on mass transfer in randomly generated porous media. The first one is called the pathpercolation model and used for random porous media generation and cluster labelling process, which are explained in the following section. Then, a single-component single-phase (SCSP) LBM was utilized to solve the momentum balance equation and obtain the velocity distribution 
in representative area elements of the randomly generated channels. As the third model, a SCMP LBM was applied to observe the effects of liquid formation on mass transfer. Furthermore, parallel processing by OpenMP was implemented to the code and a performance analysis was performed.

\subsection{Inhomogeneous Porous Media Construction}

The statistical based path-percolation theory was used to generate random inhomogeneous porous media as the first step of the current study. To start with, a confidence level study was performed to determine the necessary trial and node numbers of the simulations. A detailed analysis of confidence level studies can be found in [15, 16, 19]. In the current work, 99\% confidence level was considered, and procedure of the history number determination is as follows:

The confidence level [19] defines the reliability of any estimate:

$C L=\operatorname{Pr}\left\{\left|\frac{k}{n}-p\right| \leq \varepsilon\right\}$

In Equation (1), $p$ is the probability of a pore to occur in a node, which is called the porosity in this study. Total history number is represented by $n$, whereas $k$ stands for the number of void generation in $n$ histories. Error is defined as the difference between the true and estimated probabilities of an event, and represented by $\varepsilon$. As an example, if $\varepsilon$ is $1 \%$ for a target porosity of $90 \%$, then the resulting porosity of the statistically generated porous media would be between $89 \%$ and $91 \%$, by a $99 \%$ of probability. Finally, $\operatorname{Pr}$ in Equation (1) represents the probability of the event in the brackets, and $C L$ is the confidence level. In this study, $C L$ and $\varepsilon$ are 0.99 and 0.001, respectively. Hence, Equation (1) says that the confidence level of $99 \%$ is the probability of the difference between the estimated and the true probabilities of the porosity to be lower than or equal to the assigned error, which is 0.001 . After the application of the law of large numbers [19], Equation (2) is obtained:

$\operatorname{Pr}\left\{\left|\frac{k}{n}-p\right| \leq \varepsilon\right\}=2 \mathbb{G}\left(\varepsilon \sqrt{\frac{n}{p q}}\right)-1=C L$

In Equation (2), $q$ is the probability of generating a solid in a node, hence it is $1-p$, and $\mathbb{G}$ is the Gaussian function, and related to the error function as:

$\mathbb{G}(z)-\frac{1}{2}=\operatorname{erf}(z)$

The following equation is obtained after inserting Equation (3) into Equation (2): 


$$
\frac{C L}{2}=\operatorname{erf}\left(\varepsilon \sqrt{\frac{n}{p q}}\right)
$$

A confidence level of $99 \%$ was applied to all three cases of $\Phi=0.70, \Phi=0.80$, and $\Phi=0.90$ with the related $p$ values of $0.70,0.80$, and 0.90 , respectively. Hence, $q$ becomes $0.30,0.20$, and 0.10 . The total number of history $(n)$ is the trial number of the simulations multiplied by the total node number, and is calculated by Equation (4) and using an error function table [19]. Table 1 summarizes the confidence level results. An in-house program was developed to generate random inhomogeneous porous media by assigning random numbers to each 10,000 nodes. Then, depending on the porosity, the nodes are labeled as solid or void. The porous media generated here are considered as gas diffusion channels, and the lower and upper boundaries are accepted as inflow and outflow boundaries, respectively, while the side walls are impervious boundaries, and thus reflective.

For the next step, the cluster labeling process was performed [15, 16] by clustering the inter-connected pores. Orphaned (or isolated) clusters, which were not connected to either inflow and outflow boundaries, were eliminated and considered equivalent to a solid. The domain obtained after the cluster labeling process is called the effective domain, and the porosity of the effective domain is called the effective porosity. Thus, a single-component multiphase model can be developed now to investigate the momentum flow in the effective domains (channels) constructed by the path-percolation theory.

\subsection{The Lattice-Boltzmann Model}

The Lattice-Boltzmann Method (LBM) is a mesoscopic approach which lies between microscopic and macroscopic approaches. In LBM, individual particle tracking is not followed as in a true microscopic approach nor are the averaged properties of the entire system averaged as in a macroscopic approach. Instead, the average property of a selected cluster of molecules is used. The probability density function, $f$, must be introduced before analyzing the LatticeBoltzmann model (LBM). The probability of a molecule to be at position $\vec{x}$ at time $t$ with momentum $\vec{p}$ is called the probability density (or probability distribution) function, and symbolized as $f(\vec{x}, \vec{p}, t)$. In this study, a two-dimensional lattice system called $\mathrm{D}_{2} \mathrm{Q}_{9}$ is applied to solve the momentum balance equation as shown in Figure $1(\mathrm{a})$. $\mathrm{A}^{\mathrm{D}} \mathrm{D}_{2} \mathrm{Q}_{9}$ has nine velocity components in two dimensions. The following coupled discretized equations are used for the collision and streaming steps [1].

$f_{k}(x+c \Delta t, y+c \Delta t, t+\Delta t)-f_{k}(x, y, t)=-\frac{1}{\tau}\left[f_{k}(x, y, t)-f_{k}^{\theta q}(x, y, t)\right]$

$f_{k}(x+\Delta x, y+\Delta y, t+\Delta t)=f_{k}(x, y, t+\Delta t)$ 
$f_{k}^{e q}$ is the equivalent distribution function, and formulated for $\mathrm{D}_{2} \mathrm{Q}_{9}$ lattice as follows [3]:

$f_{k}^{\boxminus q}(x, y, t)=w_{k} \rho(x, y, t)\left[1+3 \frac{\overrightarrow{c_{k}} \times \vec{u}}{c_{s}{ }^{2}}+\frac{9}{2} \frac{\left(\overrightarrow{c_{k}} \times \vec{u}\right)^{2}}{c_{s}{ }^{4}}-\frac{3}{2} \frac{\vec{u}^{2}}{c_{s}{ }^{2}}\right]$

where, $k, \rho$, and $\overrightarrow{c_{k}}$ are lattice index ( 0 to 8 as shown in Figure 1 ), macroscopic density, and unit lattice velocity vector, respectively. The lattice speed of sound is represented by $c_{s}$ and equal to 1/3. The unit velocities of the lattice shown in Figure 1(a) can be written as:

$$
\begin{array}{lll}
c_{0, x}=0, c_{0, y}=0, & c_{1, x}=1, c_{1, y}=0, & c_{2, x}=0, c_{2, y}=1 \\
c_{3, x}=-1, c_{3, y}=0, & c_{4, x}=0, c_{4, y}=-1, & c_{5, x}=1, c_{5, y}=1 \\
c_{6, x}=-1, c_{6, y}=1, & c_{7, x}=-1, c_{7 y}=-1, & c_{8, x}=1, c_{8, y}=-1
\end{array}
$$

Furthermore, $w_{k}$ in Equation (7) is the weighting factor and defined for all lattice components as:

$$
\begin{aligned}
& w_{0}=4 / 9 \\
& w_{1}=w_{2}=w_{3}=w_{4}=1 / 9 \\
& w_{5}=w_{6}=w_{7}=w_{8}=1 / 36
\end{aligned}
$$

The lattice density, and lattice velocity components are related to the probability distribution function as shown in Equations (10) to (12).

$$
\begin{aligned}
& \rho=\sum_{k=0}^{8} f_{k} \\
& u_{x}=\frac{1}{\rho} \sum_{k=0}^{8} c_{k, x} f_{k} \\
& u_{y}=\frac{1}{\rho} \sum_{k=0}^{8} c_{k, y} f_{k}
\end{aligned}
$$

For the boundaries in Figure 1(b), bounce back boundary conditions were set on side walls, hence the dashed (unknown) vectors become equal to solid ones (computed by streaming process). The upper and lower boundaries are periodic. Thus, a single-component, single-phase $\mathrm{D}_{2} \mathrm{Q}_{9}$ model was constructed. To investigate the mass transfer in simulated porous media, a significant parameter called "effective tortuosity", $\tau_{\text {eff }}$, which is defined as the actual path travelled by a particle, divided by the shortest distance between the same points, should be computed. To do this, the velocity profile in the channel is used as follows [20]: 
$\tau_{\text {eff }}=\frac{\int_{A} u d A}{\int_{A} u_{y} d A}$

To investigate the multiphase flow, a single-component multi-phase LBM must be constructed [3, 21]. No long-range attractive forces are considered between the molecules in a single-component single-phase model. In a single-component multi-phase model, intermolecular interactions are considered and the attractive forces between the molecules cause a phase separation between liquid and vapor. Interaction between the solid surface and the fluid is not considered here and is left as a further study. In this paper, the main focus is on the effects of liquid volume on mass transfer in inhomogeneous porous media. The van der Waals equation of state (EOS), which is a non-ideal EOS, is used to account the intermolecular attractive forces in the model:

$P=\frac{n_{m} R T}{V-n_{m} b}-a\left(\frac{n}{V}\right)^{2}$

where, $T, V, n_{m}$, and $P$ are temperature, volume, number of moles, and pressure of the fluid, respectively, and $R$ is gas constant. In Equation (14), the second term on RHS accounts for intermolecular attractive forces and $n_{m} b$ represents the non-negligible volume of molecules. The following equation, that was obtained by He and Doolen [22], is used as a simpler form of the van der Waals EOS and it is applied to compute the pressure distribution along the channel:

$P=\frac{\rho}{3}+\frac{G}{6} \Psi^{2}$

where $\Psi$ and $G$ are interaction potential and interaction strength, respectively. The interaction potential is consistent in isothermal processes with the following formula [21]:

$\Psi=\Psi_{0} \exp \left(\frac{-\rho_{0}}{\rho}\right)$

where, $\Psi_{0}$ and $\rho_{0}$ are arbitrary constants. Finally, the attractive force can be computed for a $\mathrm{D}_{2} \mathrm{Q}_{9} \mathrm{LBM}$ by Equation (17).

$F=-G \Psi(x, t) \sum_{k=1}^{8} w_{k} \Psi\left(x+c_{k} d t, t\right) c_{k}$

It must be noted that the non-negligible volume term of the van der Waals EOS is neglected in this approach. It is assumed that a two-phase fluid with a nonzero thermodynamic quality percolated an inhomogeneous channel, and then a phase separation occurred due to intermolecular attractive forces as time passes. Hence, there is no phase change in the channels. 
The initial density is the controlling parameter of the two-phase system. A typical simulation is demonstrated in the results section.

The developed single-component multi-phase LBM was not applied to the entire domain with the node number of 100x100 (Table 1). Instead, a set of representative area elements (SRAE) were used as domain. A representative area element (RAE) is defined as a smaller area that represents the entire domain. The represented parameters by RAE in this study are effective porosity and tortuosity since the mass transfer is investigated. However, only the effective porosity of the entire domain is known. Hence, a single RAE may not represent the entire domain, because there is no information about the tortuosity. To increase the accuracy of the representation, a certain number of RAEs were optimally extracted from the entire domain. The term "optimally extracted" means that the effective porosities of all possible RAEs were computed, then the relative errors between those and the effective porosity of the entire domain were determined, all RAEs were ranked, and the ones with the least errors were selected. To determine the node number of a single RAE and the number of RAEs in an SRAE, confidence level studies were repeated, as presented in Table 2. In this case, the total number of histories is the multiplication of trial number, node number of an RAE, and number of RAEs in an SRAE. The details of the SRAE selection procedure can be found in [16, 23, 24]. The ensemble average of any property of an SRAE can be calculated as follows:

$\hat{X}=\frac{1}{N_{R A E}} \sum_{m=1}^{N_{R A E}} X_{m}$

In Equation (18), $X_{m}$ is any property of the $m^{\text {th }}$ element, $N_{R A E}$ is the total element number, and $\hat{X}$ is the ensemble average. Hence, a single-component multi-phase model was completed.

To investigate the diffusion behavior, a dimensionless parameter called diffusion ratio, which is the ratio of the effective $\left(D_{\text {eff }}\right)$ and bulk $\left(D_{\text {bulk }}\right)$ diffusion coefficients, must be computed. Three diffusion models were discussed in this study. Bruggeman's model [18] approximates the effective diffusion coefficient as a function of porosity as follows:

$D_{\text {eff }}=D_{b u l k} \Phi^{m}$

In this equation, the power of the porosity is caused by the tortuosity which is the denominator. Tortuosity is estimated by the following equation, and $m$ becomes 1.5 [25]:

$\tau=\Phi^{-0.5}$

Carman's model [17] includes tortuosity in addition to porosity to calculate the effective diffusion coefficient:

$D_{\text {eff }}=D_{b u l k} \frac{\Phi}{\tau}$ 
The last model discussed in this study was developed by Cekmer et al.[16], and it will be tested in this study. In this new model, the tortuosity term was modeled as:

$\tau=\Phi^{-0.6556}$

Thus, the developed effective diffusion equation becomes:

$D_{\text {eff }}=D_{\text {bulk }} \Phi_{\text {eff }}^{1.6556}$

The resulting diffusion ratios will be computed by all these models, and discussed in the results section.

\subsection{OpenMP Implementation}

An in-house program was developed to utilize the path-percolation and LatticeBoltzmann models to investigate the multi-phase mass transfer in randomly generated porous media. A simple flowchart of the serial code for a single trial is shown in Figure 2. The program reads the input parameters and starts the path-percolation model. After applying the cluster labeling process, it prints the porosity and the effective porosity of the randomly generated inhomogeneous domain, then starts the SRAE selection process. It optimally extracts 16 RAEs for an SRAE and starts LBM to solve momentum balance equation for each RAE. Single-phase results are printed and the multi-phase loop starts by increasing the controlling parameter, which is the initial density. An automated scanning system was developed, and applied to the code to obtain the exact liquid formations of $10 \%$ and $20 \%$ in the system and a critical liquid formation value where the effective porosity becomes zero (in two-dimensions), and beyond that no fluid flow occurs. Multi-phase LBM followed by cluster labelling process is applied for all initial density values until the effective porosity of the RAE reaches zero (the critical liquid formation). When the liquid formation becomes $10 \%$ or $20 \%$, the program sets up the new effective domain by treating the liquid volume that plugged the channels as solids, and then starts cluster labelling and single-phase LBM to obtain the partially saturated effective porosity and tortuosity, respectively. Then, the computations for the new RAE starts until the set number is reached. Finally, the effective porosity and the tortuosity of an SRAE is calculated by Equation (21). Furthermore, the diffusion ratio of the SRAE is calculated by three models discussed in the previous section by Equations (19), (21), and (23).

As shown in Table 2, there are 4912 simulations to perform (total trials multiplied by the number of RAEs in a set). In a serial code, only one CPU with a single thread is used. Parallelizing a code in CPU provides the execution of multiple sets of instructions (or threads) [26]. To use multi-threads in this study, OpenMP was implemented in the serial code. OpenMP is the abbreviation of Open Multi-Processing and it is a shared-memory application programming interface [27]. The parallel implementation is demonstrated in Figure 3. After the SRAE set extraction and before the LBM application on the RAEs, an OpenMP FOR directive was implemented to allow multi-threads to work simultaneously. In Figure 3, in the upper red box, the term "chunk" stands for the number of tasks assigned per thread. Hence, each thread is assigned for one RAE (chunk=1). The command "dynamic" makes a thread be dynamically assigned to another RAE after completing one. If "static" assignment was selected, then the total 
number of the RAEs would be divided by the thread number and all the threads would be assigned to equal number of RAEs. A dynamic for loop was applied in this implementation. The fork-joint model of OpenMP with four threads is also demonstrated in the figure. The program starts with a single thread, called master thread. When a parallel region starts, the master thread generates a specified number of threads. All the threads except the master thread are terminated at the end of the parallel region.

Speedup $(S)$, which is defined as the ratio of the runtime with a single processor $\left(T_{1}\right)$ to the runtime of the parallel configuration with $n_{c}$ threads $\left(T_{n_{e}}\right)$, is used in performance analysis of parallel implementation [26]:

$S=\frac{T_{1}}{T_{n_{C}}}$

Another parameter to demonstrate the performance of the parallel code is the parallel efficiency, which is determined by dividing the speedup by the number of threads [27]:

$\varepsilon_{\text {parallel }}=\frac{T_{1}}{n_{c} T_{n_{e}}}$

To comment on the performance of the parallelized code, it will be useful to compare it with the peak performance of the used hardware. A personal computer with 4 processors with $2.40 \mathrm{GHz}$ speed, each manages 4 instructions per cycle, in other words 4 floating-point operation (Flop) per cycle was used here. Hence, the peak performance becomes:

$P_{\text {peak }}=\frac{2.40 \mathrm{GHz}}{1 \text { core }} \times \frac{4 \text { Flop }}{\text { cycle }} \times 4$ cores $=38.4 \frac{\text { GFlop }}{\mathrm{sec}}$

To calculate the performance of the code, total floating-point operations, which is a function of RAE effective porosity and number of multi-phase iterations per RAE, must be determined and divided by the runtime. The resulting number of the total floating-point operations in the code is calculated below:

$N=5.55 \times 10^{9} \sum_{i=1}^{16} \Phi_{\text {eff }}(i)[\operatorname{ITER}(\mathrm{i})+3.4145]+8.0128 \times 10^{4}+3 \sum_{i=1}^{16} \operatorname{ITER}(\mathrm{i})$

In Equation (27), ITER (i) is the iteration number for RAE number $i$ where $i=1,2, \ldots, 16$. In this equation, the second and the third terms do not contribute to the result, hence they are considered negligible. The resulting performance equation is shown in Equation (28).

Performance $=5.55 \times 10^{3} \sum_{i=1}^{16} \Phi_{\text {eff }}(i)[\operatorname{ITER}(\mathrm{i})+3.4145] /$ Execution Time 
A performance application programming interface (PAPI) timer [28] was used to measure the execution time. Three simulations per each porosity level were performed with different thread numbers, and the results of the parallel program performance were analyzed in the following section.

\section{Results and Discussion}

The first step of this study is the confidence-level analysis, which is the beginning part of the statistical based path-percolation theory. As seen in Table 1, porous media with 70\%, 80\%, and $90 \%$ porosity and 100x100 nodes were generated, and the calculated trial numbers with $99 \%$ confidence level are 140, 107, and 60, respectively. Before discussing the confidence level results, a typical trial with an $80 \%$ of target porosity is explained here. In this example, a random inhomogeneous porous medium was generated as seen in Figure 4(a) with a porosity of 0.809. Then, cluster labelling process was performed to group the interconnected pores, and eliminate the orphan pore clusters. The orphan clusters are presented in Figure 4(b). The effective domain was obtained after treating the orphan clusters as solid nodes as seen in Figure 4(c). The effective domain is now considered as a porous flow channel with an inlet at the bottom and an outlet at the top boundaries. The effective porosity was computed as 0.796 . The next step is the SRAE extraction. The program optimally extracted 16 RAEs from the effective domain. Three samples are shown in Figure 4(d)-(f). The effective porosities of all of the RAEs can be seen in Figure 5(a). Then, a single-component single-phase LBM was applied to obtain the velocity distribution in these 16 domains and the effective tortuosities were computed as seen in Figure 5(b). The same results were also demonstrated in Table 3 with an addition of effective porosity and tortuosity calculated for SRAE by Equation (18), and the diffusion ratios by three different models. It can be concluded that the Carman model and the new model overlap within this effective porosity range (about $80 \%$ ). The results for REV \#2 were also analyzed. The effective domain of this representative area can be seen in Figure 4(d). The path of the fluid particles was obtained after the application of single-phase LBM as shown in Figure 6. The tortuosity was computed as 1.153 whereas the effective porosity was 0.8 . The effects of liquid formation on effective porosity and tortuosity are discussed next.

A multi-phase LBM was applied to the 16 RAEs of the domain of the example. At this time, a portion of the percolated fluid was assumed to be liquid particles, and started to merge due to intermolecular attractive forces. The time evolution of this intermolecular attraction of a $10 \%$ condensed fluid in RAE \#2 is shown in Figure 7 . It must be noted that all the units of the lattice parameters are called the lattice units and the relation to the SI units can be found in [1, 3, 4]. After 5 lattice seconds ( $l s$ ) liquid and vapor phases started to separate from each other and the liquid particles clustered together as time passes. After 20,000 $l s$ the system reached steady-state. To analyze the vapor flow in the channel, single-phase LBM must be applied. Before that, the system was set up by considering the liquid clusters as solid, since they plugged some of the void nodes. Then, the cluster labelling process was again performed to obtain the new effective domain with $10 \%$ condensate, as seen in Figure 8. Liquid formation is shown in gray in Figure 8(b) and they were considered solid as were orphan pore clusters. The effective domain with an 
effective porosity of 0.714 can be seen with the velocity field of the molecules in vapor phase in Figure 8(c). The effective tortuosity is observed to be increased to 1.310 when the condensate was up to $10 \%$. Figure 5(c) and (d) shows the effective porosities and tortuosities of all RAEs with a condensate of $10 \%$, respectively. The effective porosity values decreased about $10 \%$ and a slight increase in tortuosity levels are observed. Similarly, 20\% liquid formation effects are shown in Figure 9. The effective porosity and tortuosity are 0.584 and 1.552, respectively. In Figure 5(e) and (f), all the RAE parameters are demonstrated by a bar plot and the SRAE properties with diffusion ratio results are shown in Figure 10 (a) and (b), respectively.

The previous example was a single trial with $80 \%$ porosity. As seen in Table 1, 140, 107, and 60 trials were completed for $70 \%, 80 \%$, and 90\% porosity, respectively, each with one SRAE constituted by 16 RAEs. The results for the porosities and the effective porosities of the initially generated random porous media are shown in Figure 11. In this figure, probability distributions for both porosities and effective porosities for the media with all three target porosities with a confidence level of $99 \%$ were plotted. The results for all trials were divided into subgroups and these subgroups were plotted versus their frequency of occurrence. It is observed that all six curves have Gaussian shapes. For $90 \%$ target porosity, the effective porosity and porosity probability curves almost coincide. When the target porosity decreases, the effective porosity - probability distribution curve becomes wider in range, and shifts to the left.

Figure 12 shows the effects of liquid formation on effective porosity and tortuosity. In Figure 12(a), the results for $90 \%$ target porosity is shown. Effective porosity was decreased by increased liquid formation in the channel and the effective porosity probability distribution range was increased. Furthermore, the occurrence frequency was also decreased. Similar results were observed for the other target porosity cases, Figure 12(b) for $80 \%$, and (c) for $70 \%$ target porosity, respectively, only the distribution ranges got wider, and the occurrence frequency was decreased with target porosity. Interestingly, the effective tortuosity results do not show any monotonic trend with the impact of liquid formation on effective porosity, especially for the target porosity of $70 \%$ (Figure 12(c)). To start with, as the target porosity decreases the tortuosity in dry media and the probability distribution range of tortuosity increase. For a target porosity of $80 \%$ and $90 \%$, the liquid formation increases the tortuosity (Figure 12 (a) and (b)) as in the example for $80 \%$ target porosity discussed above (Figures 4 to 10). For $70 \%$ target porosity, the results do not follow this trend for $20 \%$ condensate, as seen in Figure 12 (c). For $10 \%$ liquid formation, the tortuosity probability distribution curve slightly shifts to the right and the width of the curve slightly increases. For $20 \%$ liquid formation, the curve shifts to the left and gets wider in range. To understand this behavior, many trials were examined, one of which is discussed here. The trial had a target porosity of $70 \%$ and the resulting porosity and effective porosity values are 0.701 and 0.687 , respectively. Multi-phase effects in RAE \#1 were studied, and are shown in Figures 13 and 14. In Figure 13(a) and (b), 10\% liquid formation is observed and the cluster labelling was applied to the figures on the right. The domains in Figure 14 are the effective domain obtained after cluster labelling process. In Figure 14(a), the velocity field in a dry domain is shown. The computed effective porosity and tortuosity are 0.686 and 1.403 , respectively. With $10 \%$ liquid formation, a portion of the channel at bottom left was closed by liquid clusters, and a small portion of that was constituted by orphan clusters, hence eliminated 
and considered as a solid by the cluster labelling process. Then, tortuosity increased to 1.448 whereas the effective porosity decreased to 0.576 . The related velocity field can be seen in Figure 14 (b). With $20 \%$ condensate, the liquid clusters from the previous case with $10 \%$ condensate widened and a new liquid cluster occurred as seen in Figure 13 (b). The velocity field in the effective domain for this case can be seen in Figure 14 (c). After comparing the paths of the molecules between dry and $20 \%$ liquid cases, it is observed that two main paths (one on the left and one at the center) were eliminated as seen in Figure 14 (c). Hence, the most of the molecules travel from the right part of the channel with a tortuosity of 1.286. The effective porosity in this case is 0.522 . It can be concluded that the paths eliminated by liquid formation decreased the average effective tortuosity. Although a reduced tortuosity improves the mass transfer [17], the effective diffusion coefficient can still decrease, because of the changes in the effective porosity, which have a great impact on mass transfer. Figure 15 shows the effects of liquid formation on diffusion ratio in porous media with a target porosity of $70 \%$ for three diffusion models. As observed in the figure, diffusion ratios calculated by all three model equations decreased with increasing liquid formation in porous channels and the probability distribution curves of diffusion ratio got wider in range. Furthermore, it can be seen that the diffusion ratio calculated by the Carman model differed significantly from the other models in media with $20 \%$ liquid formation. The reason is clearly the tortuosity effect. From Figure 15, it can be concluded that the effects of tortuosity become more important in mass transfer, when the liquid formation increases and the effective porosity decreases. Three-dimensional simulations would also attest this conclusion, since the tortuosity values will definitely increase with the addition of the third dimension.

The critical liquid formation is another consideration of mass transfer. When the liquid formation reaches a critical value, where the effective porosity becomes zero, mass transfer is shut down in a channel. Figure 16 shows the critical liquid formation probability distributions for all target porosities. For $90 \%$ target porosity, critical liquid formation had values between 0.5 and 0.8 , and the range was approximately 0.3 , as seen in the figure. The maximum occurrence frequency is $22 \%$ and the related critical liquid formation is around $70 \%$. Critical liquid formation probability range increases significantly with decreased target porosity. The maximum occurrence frequency for $80 \%$ porosity is about $20 \%$ and the most encountered critical liquid formation values are around 45\%. The critical liquid formation has values between 0.32 and 0.69 , hence the range is 0.37 , which is $7 \%$ greater than that for $90 \%$ target porosity. For $70 \%$ target porosity, the critical liquid formation has values between 0.16 and 0.57 , hence the probability distribution range is 0.41 , which is $4 \%$ and $11 \%$ larger than the cases with $80 \%$ and $90 \%$ target porosity. On the other hand, the maximum occurrence frequency reduced to $16 \%$ for around $32 \%$ critical liquid formation.

OpenMP was implemented to the code and the plots for speedup, parallel efficiency, and performance of the simulations for three domains with $70 \%, 80 \%$, and $90 \%$ target porosity can be seen in Figure 17. The maximum performance was obtained with 4 threads for the channel with $90 \%$ porosity as 1.275 GFlops, which is $2.6 \%$ of the peak performance. The related speedup and efficiency were computed 3.355 and $83.88 \%$, respectively. The performance and speedup decreased with reduced target porosity. The reason of this result is that the total iteration number 
also decreases with the target porosity, since the program finds the critical liquid formation faster. It is concluded that the change in iteration number gets greater than the change in execution time (Equation 28), when target porosity increases. The best option with the specified confidence level study is using 16 physical cores, and assigning 16 threads, since there are 16 RAEs in each trial.

This model is a two-dimensional approximation to a multi-phase problem in a porous medium and there are several sources of potential error. The surface-liquid interaction effects were neglected. Furthermore, the neglected third dimension can cause a loss of accuracy, since the effective tortuosities are underestimated in two-dimensional simulations, as the potential paths into the third dimension are not included. The effective porosities are also underestimated, since there would be additional paths, which would decrease the number of orphan pore clusters in three-dimensional simulations. The viscous coupling effects between gas and liquid phases were also not considered. For applications with low relative velocities of the phases, this effect should be negligible. However, the results do demonstrate the ability of this unique approach to simulate multiphase flow in porous media. Moreover, an experimental validation is needed for this computational study. On the other hand, an improvement on parallel application can be performed by utilizing an alternative hybrid parallel model to increase the speedup, efficiency, and performance. These missing physics are the subject of the ongoing study.

\section{Conclusions}

In this study, the path-percolation theory and Lattice-Boltzmann method were utilized to develop a model to investigate the effects of liquid formation on gas-phase mass transfer in porous media. A statistical study was performed to analyze the behavior of effective porosity and tortuosity under different solid-liquid-vapor configurations of the media with three different porosities. The results show that the effective porosity decreases when the liquid formation in a channel increases. The impact of liquid formation on effective tortuosity changes with porosity, and it can decrease or increase with liquid formation. However the effective diffusion coefficient decreases with liquid formation. Furthermore, critical liquid formation was investigated for all three media, and its value increased with target porosity. On the other hand, when the target porosity decreases, it becomes hard to predict the critical liquid formation, since the range of the possible results gets wider. Finally, OpenMP was implemented to the model code and a performance analysis was applied using multi threads. The observed maximum speedup was 3.3553 with a performance of 1.275 GFlops using four threads.

The results of this study can be used for porous media applications related to two-phase mass transfer, and is expected to provide an alternative statistical based approach to estimate the effective porosity, tortuosity, and diffusion ratio in porous media with a wide range of porosity and liquid formation. Extension to three dimensions is computationally more intensive, but straight-forward analytically. 


\section{Disclaimer}

This report was prepared as an account of work sponsored by an agency of the United States Government. Neither the United States Government nor any agency thereof, nor any of their employees, makes any warranty, express or implied, or assumes any legal liability or responsibility for the accuracy, completeness, or usefulness of any information, apparatus, product, or process disclosed, or represents that its use would not infringe privately owned rights. Reference herein to any specific commercial product, process, or service by trade name, trademark, manufacturer, or otherwise does not necessarily constitute or imply its endorsement, recommendation, or favoring by the United States Government or any agency thereof. The views and opinions of authors expressed herein do not necessarily state or reflect those of the United States Government or any agency thereof.

\section{Acknowledgment}

This material is based upon work supported by the Department of Energy under Award Number DE-EE0000470.

\section{References}

[1] S. Succi, The Lattice Boltzmann Equation for Fluid Dynamics and Beyond, Clarendon Press; Oxford University Press, Oxford New York, 2001.

[2] L. Boltzmann, Lectures on Gas Theory, University of California Press, Berkeley, 1964.

[3] M.C. Sukop, D.T. Thorne, Lattice Boltzmann Modeling: An Introduction for Geoscientists and Engineers, Springer, Berlin ; New York, 2006.

[4] A.A. Mohamad, Lattice Boltzmann Method: Fundamentals and Engineering Applications with Computer Codes, Springer-Verlag, London, 2011.

[5] M.C. Sukop, H.B. Huang, P.F. Alvarez, E.A. Variano, K.J. Cunningham, Evaluation of permeability and non-Darcy flow in vuggy macroporous limestone aquifer samples with lattice Boltzmann methods, Water Resour Res, 49 (2013) 216-230.

[6] X.P. Li, Y. Zhang, X.W. Wang, W. Ge, GPU-based numerical simulation of multi-phase flow in porous media using multiple-relaxation-time lattice Boltzmann method, Chem Eng Sci, 102 (2013) 209-219.

[7] H.B. Huang, J.J. Huang, X.Y. Lu, M.C. Sukop, On simulations of high-density ratio flows using color-gradient multiphase lattice Boltzmann Models, Int J Mod Phys C, 24 (2013) 1350021.

[8] A. Genty, V. Pot, Numerical simulation of 3D liquid-gas distribution in porous media by a two-phase TRT Lattice Boltzmann method, Transport Porous Med, 96 (2013) 271-294.

[9] H. El Abrach, H. Dhahri, A. Mhimid, Numerical simulation of drying of a saturated deformable porous media by the Lattice Boltzmann method, Transport Porous Med, 99 (2013) 427-452.

[10] H.B. Huang, Z.T. Li, S.A.S.A. Liu, X.Y. Lu, Shan-and-Chen-type multiphase lattice Boltzmann study of viscous coupling effects for two-phase flow in porous media, Int J Numer Meth Fl, 61 (2009) 341-354.

[11] H.B. Huang, L. Wang, X.Y. Lu, Evaluation of three lattice Boltzmann models for multiphase flows in porous media, Comput Math Appl, 61 (2011) 3606-3617. 
[12] M.C. Sukop, H. Huang, C.L. Lin, M.D. Deo, K. Oh, J.D. Miller, Distribution of multiphase fluids in porous media: Comparison between lattice Boltzmann modeling and micro-X-ray tomography, Phys Rev E, 77 (2008) 026710.

[13] J.F. Chau, D. Or, M.C. Sukop, Simulation of gaseous diffusion in partially saturated porous media under variable gravity with lattice Boltzmann methods, Water Resour Res, 41 (2005) W08410.

[14] J.F. Chau, D. Or, M.C. Sukop, Simulation of gaseous diffusion in partially saturated porous media under variable gravity with lattice Boltzmann methods, Water Resour Res, 41 (2005).

[15] H.M. Jung, W. Choi, S. Um, Path-percolation modeling of the electrical property variations with statistical procedures in spatially-disordered Inhomogeneous media, J Korean Phys Soc, 56 (2010) 591-597.

[16] O. Cekmer, S. Um, M.M. Mench, Application of path-percolation theory and LatticeBoltzmann method to investigate structure-property relationships in porous media, Int $\mathbf{J}$ Heat Mass Tran, 86 (2015) 101-112.

[17] P.C. Carman, Flow of gases through porous media, Butterworth's Scientific Publications, London, 1956.

[18] D.A.G. Bruggeman, Calculation of various physics constants in heterogenous substances I Dielectricity constants and conductivity of mixed bodies from isotropic substances, Annalen Der Physik, 24 (1935) 636-664.

[19] A. Papoulis, Citation Classic - Probability, Random-Variables, and Stochastic-Processes, Cc/Eng Tech Appl Sci, (1980) 14-14.

[20] M. Matyka, A. Khalili, Z. Koza, Tortuosity-porosity relation in porous media flow, Phys Rev E, 78 (2008) 026306.

[21] X.W. Shan, H.D. Chen, Simulation of nonideal gases and liquid-gas phase-transitions by the Lattice Boltzmann-equation, Phys Rev E, 49 (1994) 2941-2948.

[22] X.Y. He, G.D. Doolen, Thermodynamic foundations of kinetic theory and Lattice Boltzmann models for multiphase flows, J Stat Phys, 107 (2002) 309-328.

[23] E.A. Wargo, A.C. Hanna, A. Cecen, S.R. Kalidindi, E.C. Kumbur, A representative volume element approach for pore-scale modeling of fuel cell materials, Polymer Electrolyte Fuel Cells 11, 41 (2011) 131-139.

[24] E.A. Wargo, A.C. Hanna, A. Cecen, S.R. Kalidindi, E.C. Kumbur, Selection of representative volume elements for pore-scale analysis of transport in fuel cell materials, Journal of Power Sources, 197 (2012) 168-179.

[25] D.W. Chung, M. Ebner, D.R. Ely, V. Wood, R.E. Garcia, Validity of the Bruggeman relation for porous electrodes, Model Simul Mater Sc, 21 (2013) 074009.

[26] J.J. Dongarra, Sourcebook of Parallel Computing, Morgan Kaufmann Publishers, San Francisco, CA, 2003.

[27] B. Chapman, G. Jost, R.v.d. Pas, Using OpenMP: Portable Shared Memory Parallel Programming, MIT Press, Cambridge, Mass., 2008.

[28] S. Browne, J. Dongarra, N. Garner, G. Ho, P. Mucci, A portable programming interface for performance evaluation on modern processors, International Journal of High Performance Computing Applications, 14 (2000) 189-204. 
Table 1 - History calculations for path-percolation model.

\begin{tabular}{|ccrrrrr|}
\hline $\begin{array}{c}\text { Confidence } \\
\text { Level }\end{array}$ & $\operatorname{erf}\left(\varepsilon \sqrt{\frac{n}{p q}}\right)$ & $\varepsilon \sqrt{\frac{n}{p q}}$ & Nodes & $\phi$ & $n$ & Trials \\
\hline $99 \%$ & 0.495 & 2.5767 & $100 \times 100$ & 0.80 & $1,062,301$ & 107 \\
& & & 0.90 & 597,544 & 60 \\
\hline
\end{tabular}

[Type here] 
Table 2 - History calculations for Lattice-Boltzmann model.

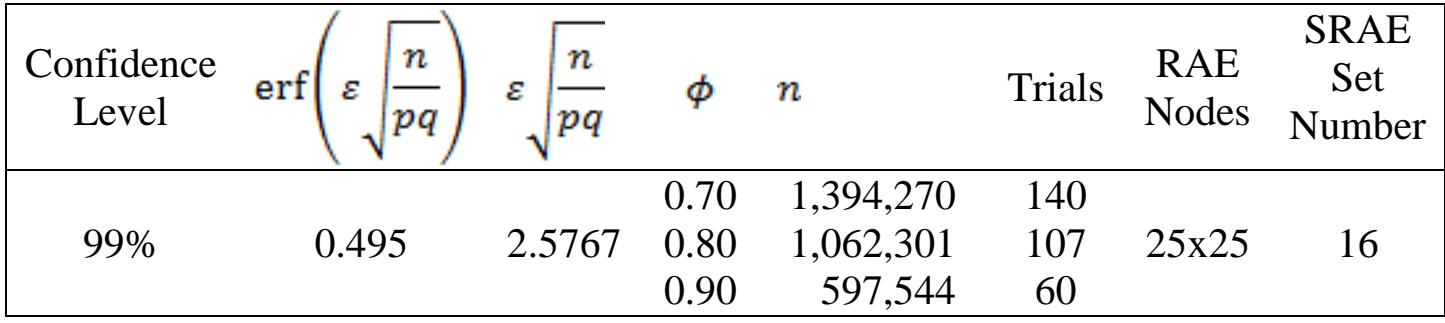


Table 3 - Effective porosity, tortuosity, and diffusion ratio calculation example for SRAE with $0 \%$ condensate.

\begin{tabular}{|cccccccc|}
\hline RAE \# & $\phi_{\text {eff }}$ & $\tau_{\text {eff }}$ & $\phi_{\text {eff }, \text { SRAE }}$ & $\tau_{\text {eff }, \text { SRAE }}$ & $Q_{\text {Bruggeman }}$ & $Q_{\text {Carman }}$ & $Q_{\text {NewModel }}$ \\
\hline 1 & 0.795200 & 1.150737 & & & & \\
2 & 0.800000 & 1.153163 & & & & \\
3 & 0.801600 & 1.154434 & & & & \\
4 & 0.798400 & 1.157320 & & & \\
5 & 0.793600 & 1.155720 & & & \\
6 & 0.798400 & 1.162446 & & & & \\
7 & 0.796800 & 1.173685 & & & & \\
8 & 0.792000 & 1.161168 & 0.795200 & 1.164617 & 0.709112 & 0.682800 & 0.684272 \\
9 & 0.795200 & 1.130812 & & & & \\
10 & 0.798400 & 1.161545 & & & & \\
11 & 0.792000 & 1.169265 & & & & \\
12 & 0.788800 & 1.170493 & & & & \\
13 & 0.788800 & 1.175958 & & & & \\
14 & 0.790400 & 1.177900 & & & & \\
15 & 0.792000 & 1.196414 & & & & \\
16 & 0.801600 & 1.182811 & & & & & \\
\hline
\end{tabular}




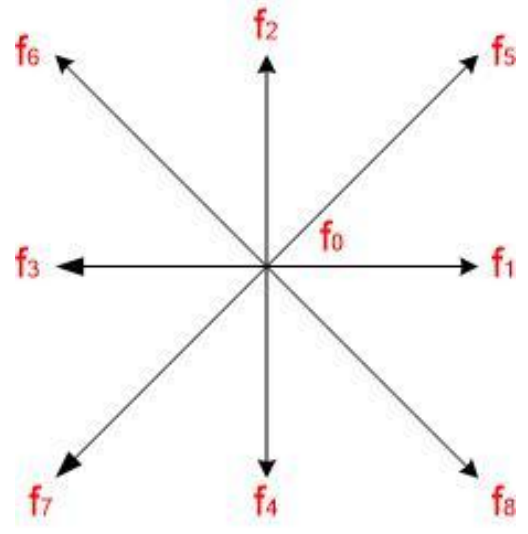

(a)

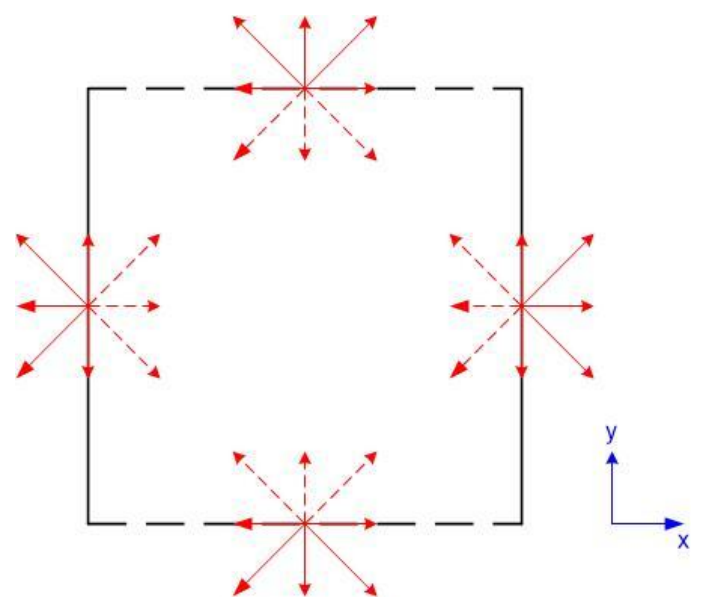

(b)

Figure $1-\mathrm{A} \mathrm{D}_{2} \mathrm{Q}_{9}$ lattice (a) a lattice and its velocity components (b) red arrows are the lattice velocities at boundaries; the dashed ones are unknown. 
Figure 2

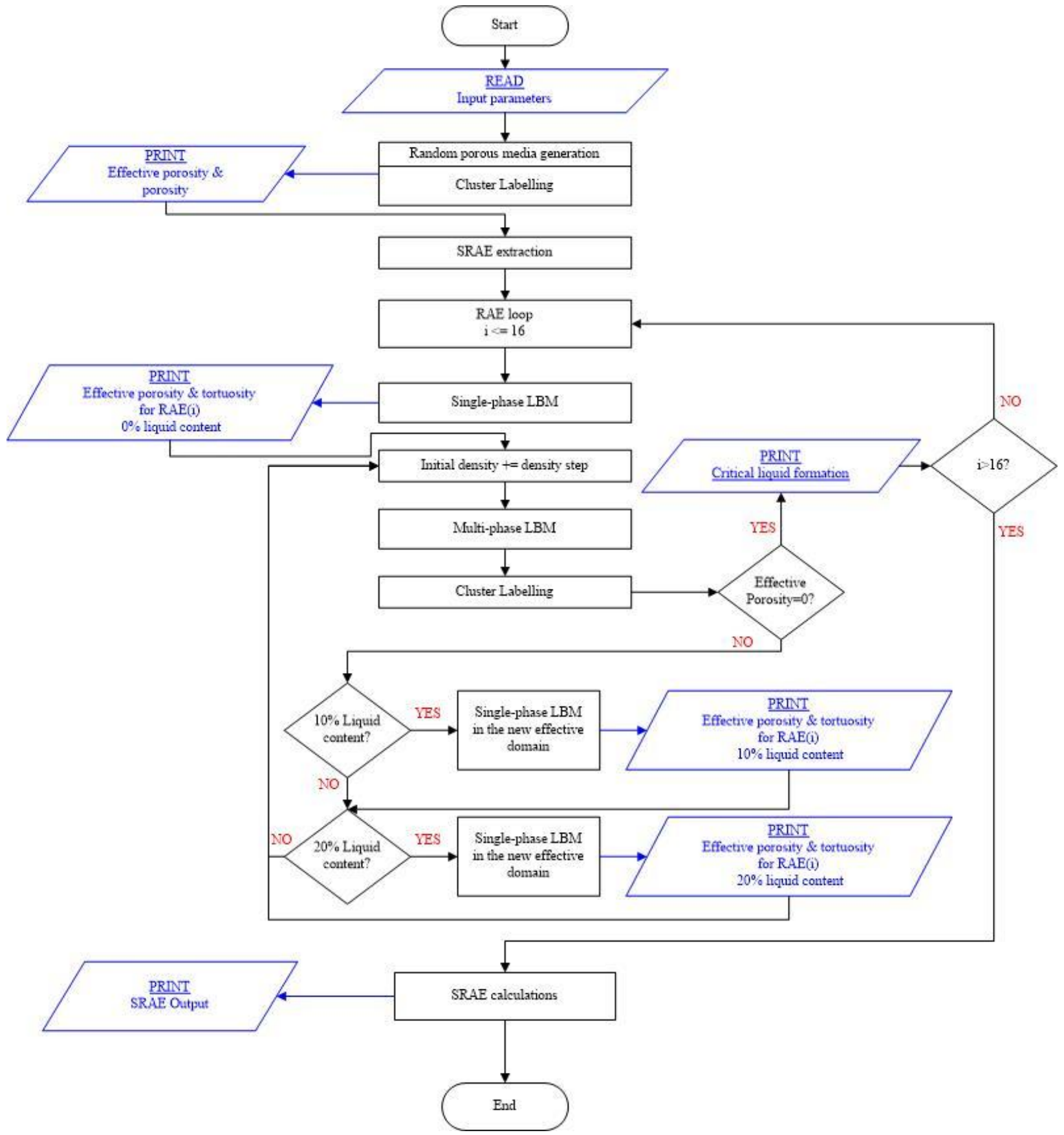

Figure 2 - Flowchart of the serial code for a single trial. 
Figure 3

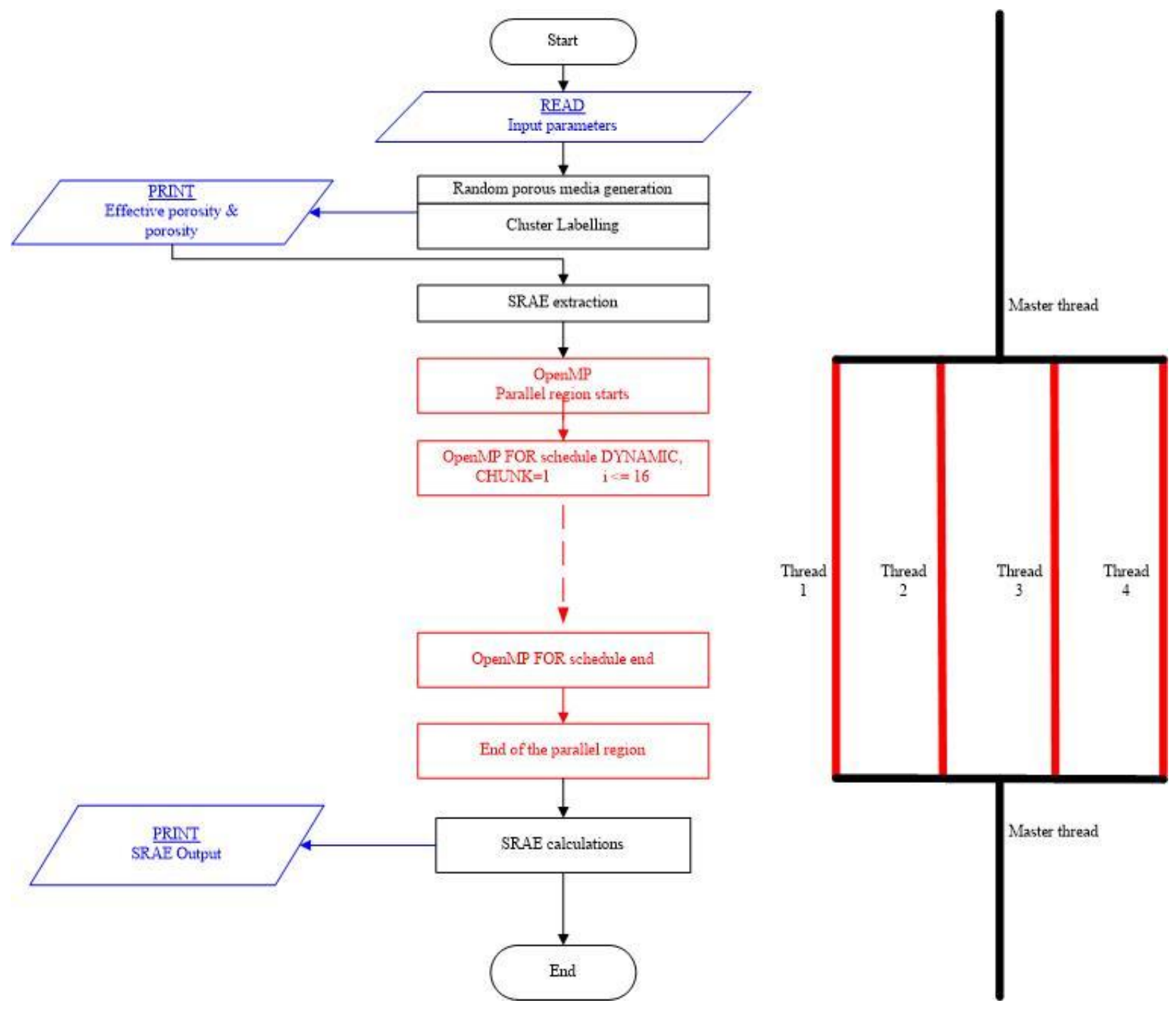

Figure 3 - OpenMP implementation to the serial code. Flowchart of the parallel code for a single trial and demonstration of the fork-join model with 4 threads. 


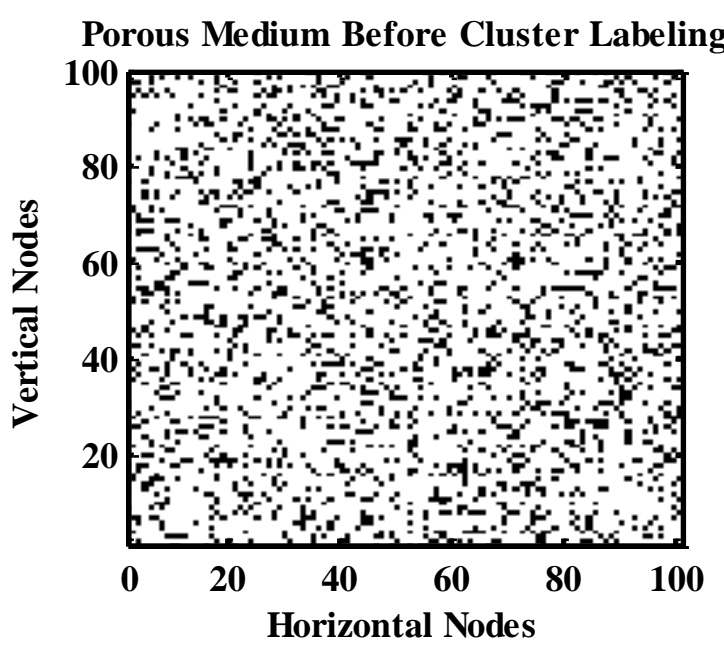

(a)

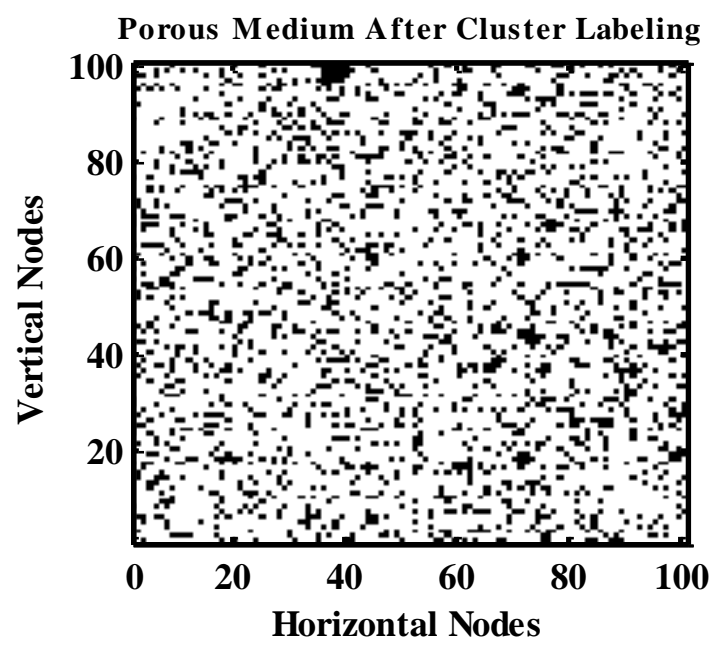

(c)

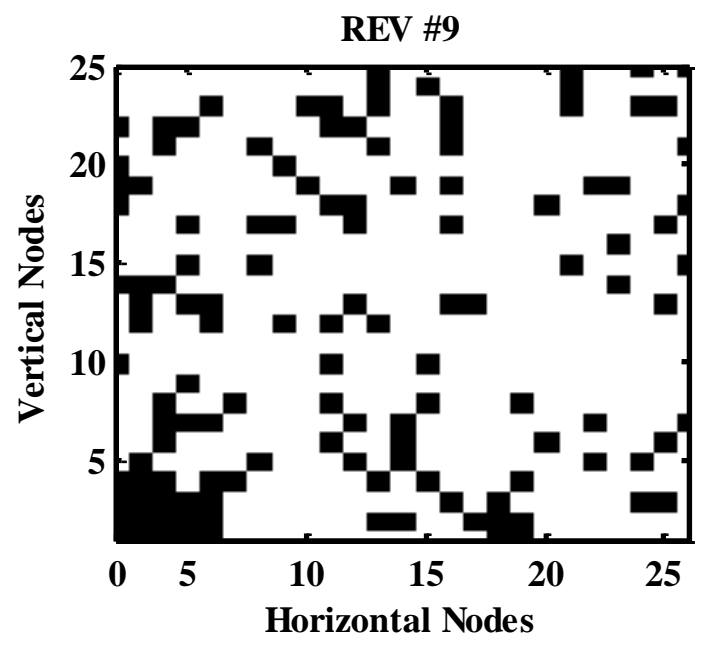

(e)

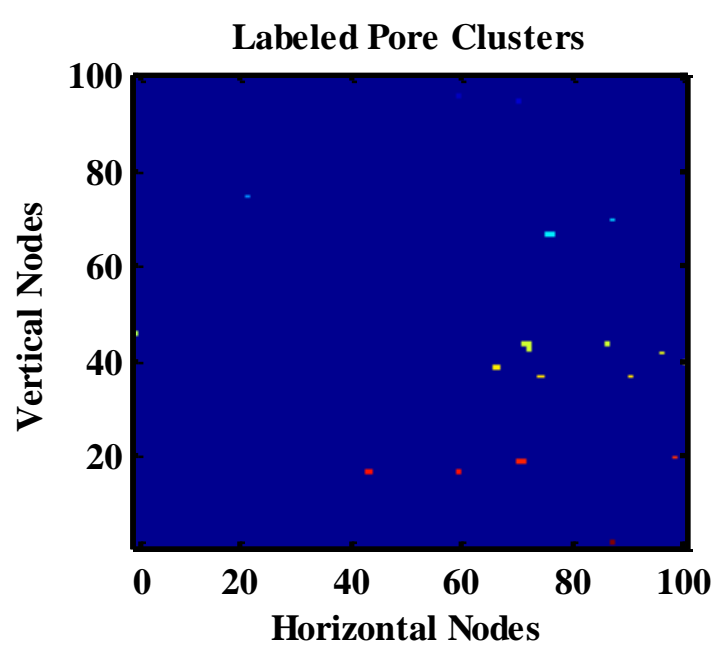

(b)

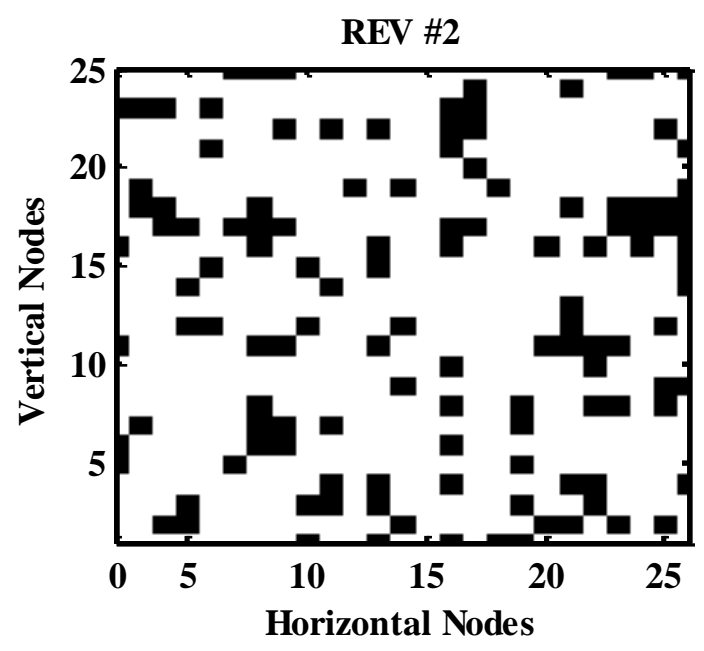

(d)

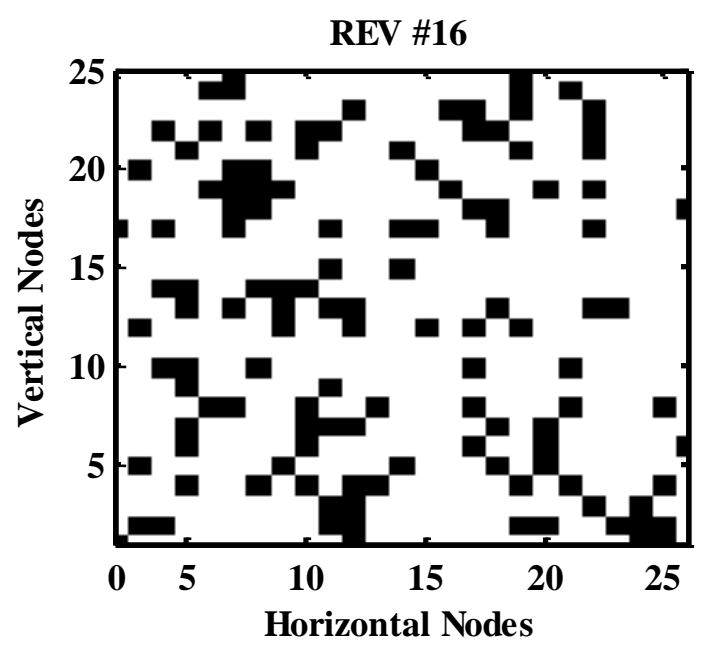

(f) 
Figure 4 - Path-percolation model for initial porous medium generation (a) domain before cluster labelling (b) cluster labelling (c) effective domain (d) - (f) three random RAEs. 


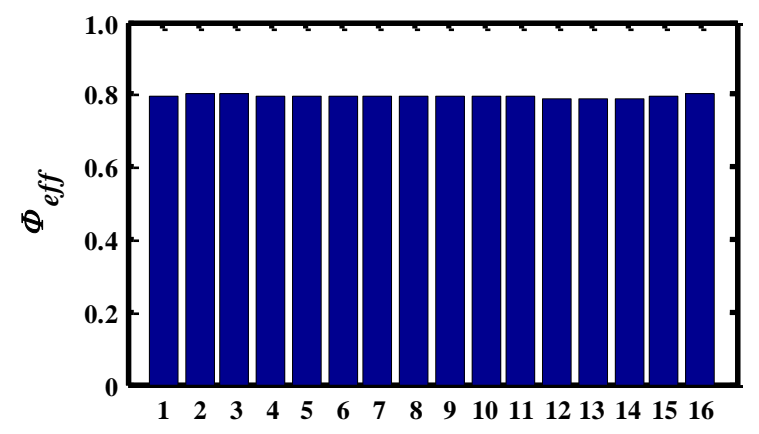

Representative area elements

(a)

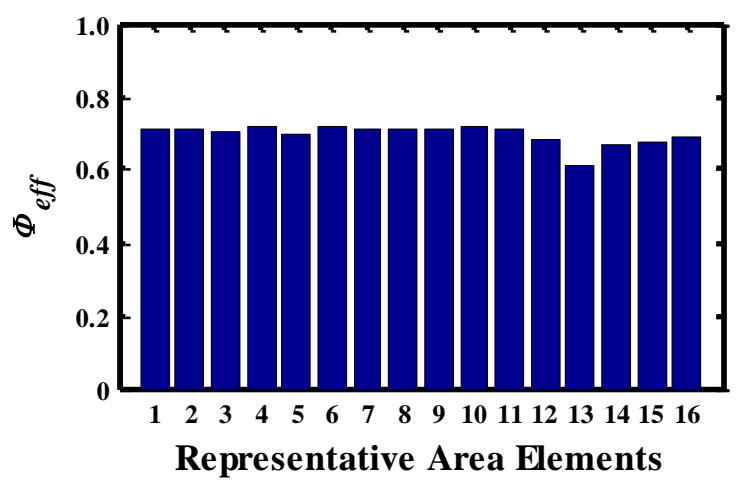

(c)

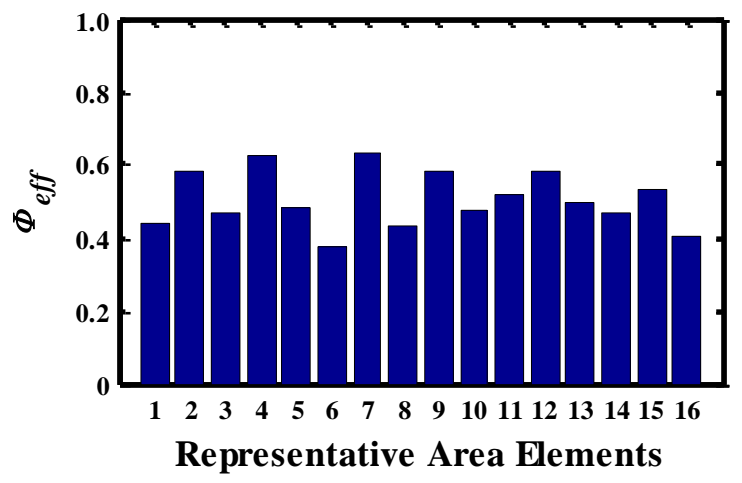

(e)

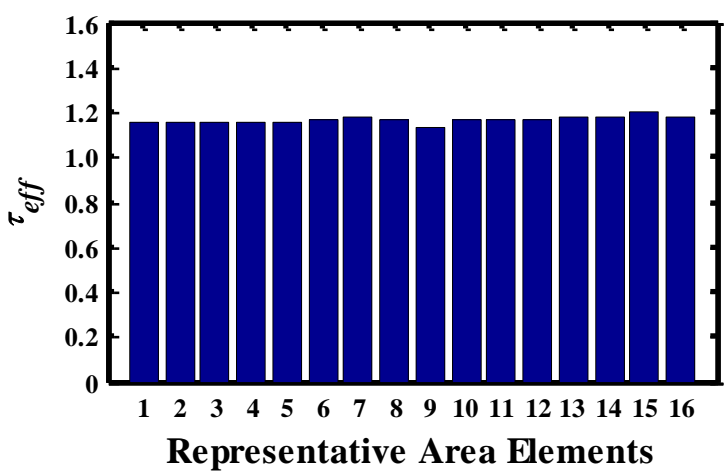

(b)

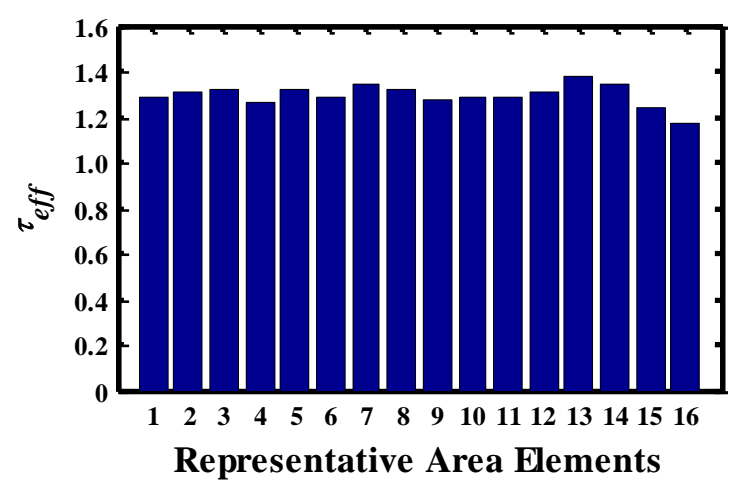

(d)

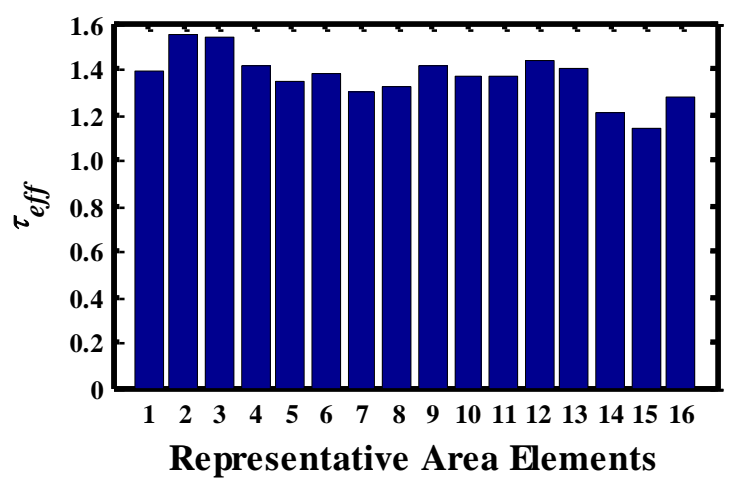

(f)

Figure 5 - Effective porosity \& tortuosity calculations for RAEs of an example trial with $80 \%$ porosity and $0 \%$ condensate (a) $\Phi_{\text {eff }}$ with $0 \%$ condensate (b) $\tau_{\text {eff }}$ with $0 \%$ condensate (c) $\Phi_{\text {eff }}$ with $10 \%$ condensate (d) $\tau_{\text {eff }}$ with $10 \%$ condensate (e) $\Phi_{\text {eff }}$ with $20 \%$ condensate (f) $\tau_{\text {eff }}$ with $20 \%$ condensate. 
Figure 6

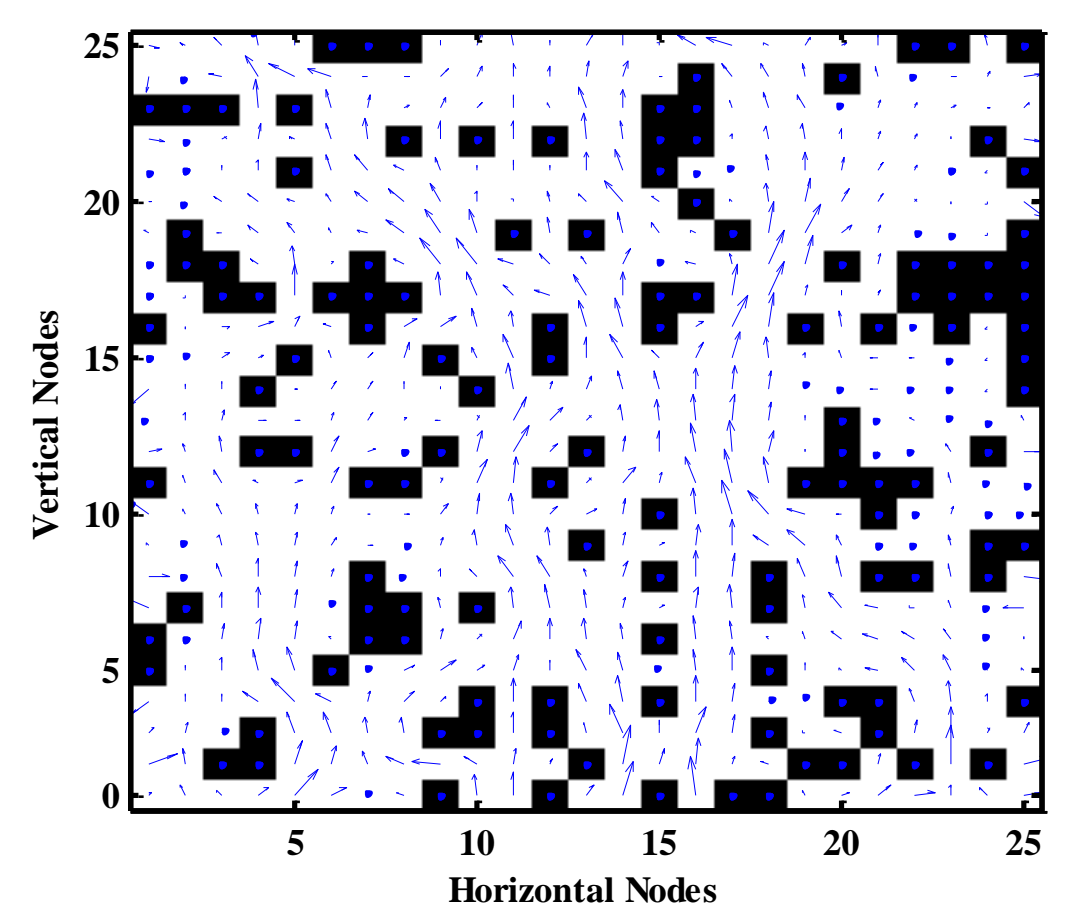

Figure 6 - Velocity vectors along a representative porous domain, RAE \#2. Tortuosity is 1.153.

Horizontal Nodes 

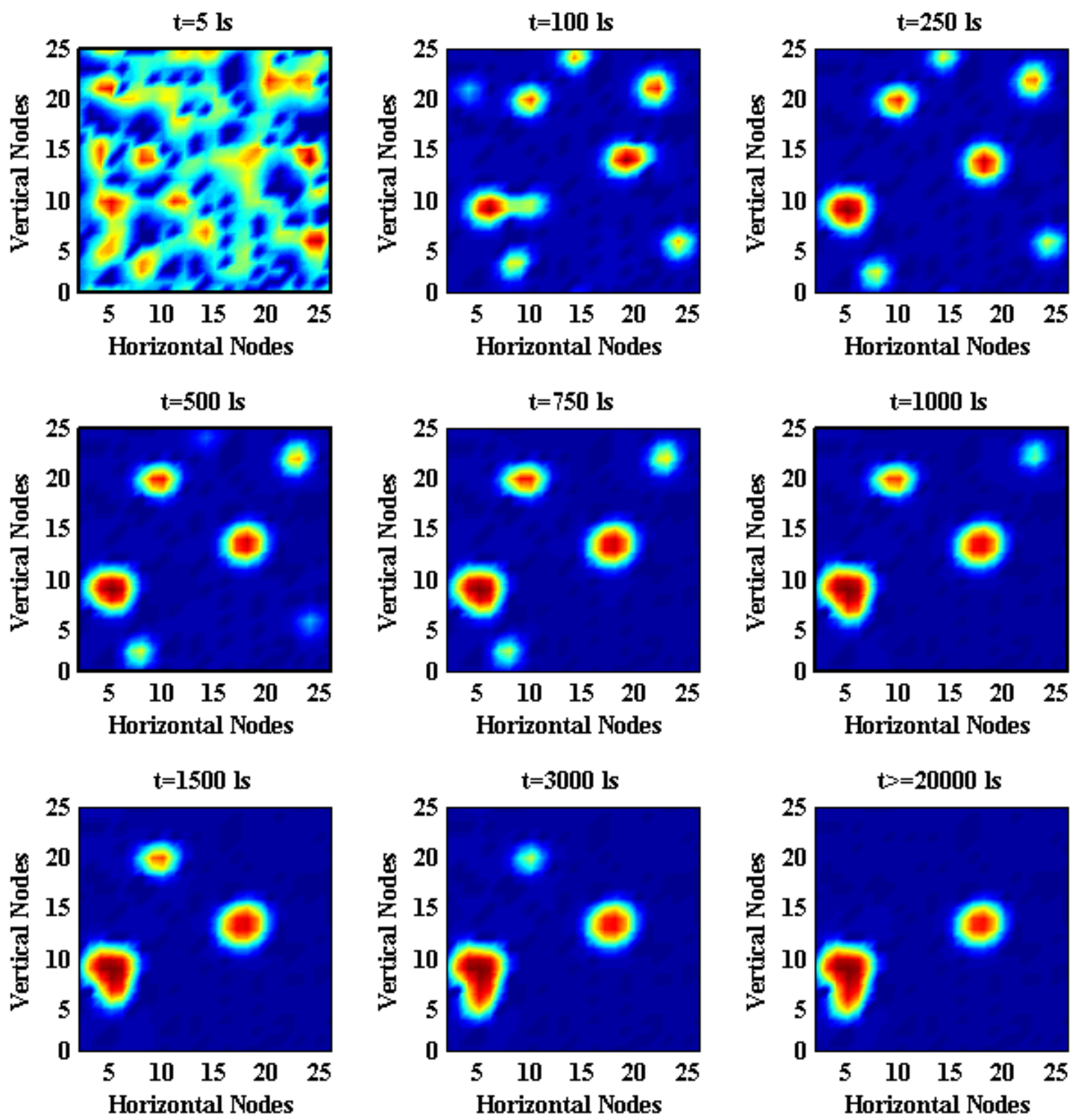

Figure 7 - Evolution of phase separation of a two-phase fluid with a $10 \%$ condensate in REV \#2. 


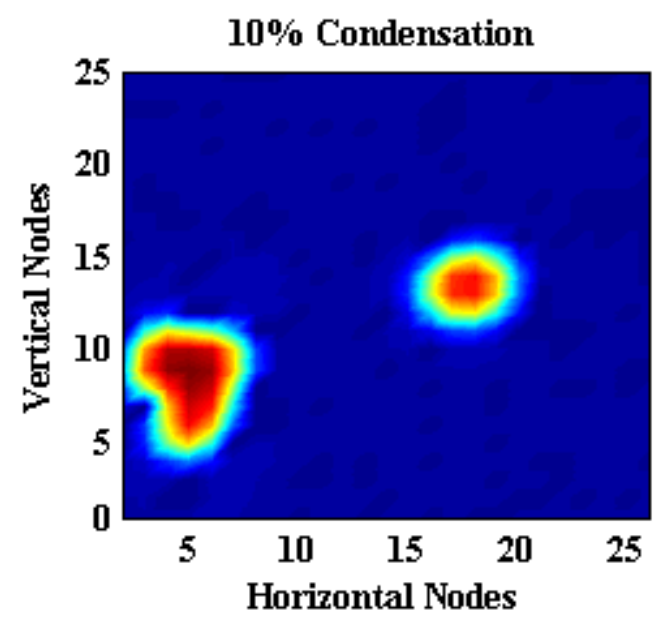

(a)

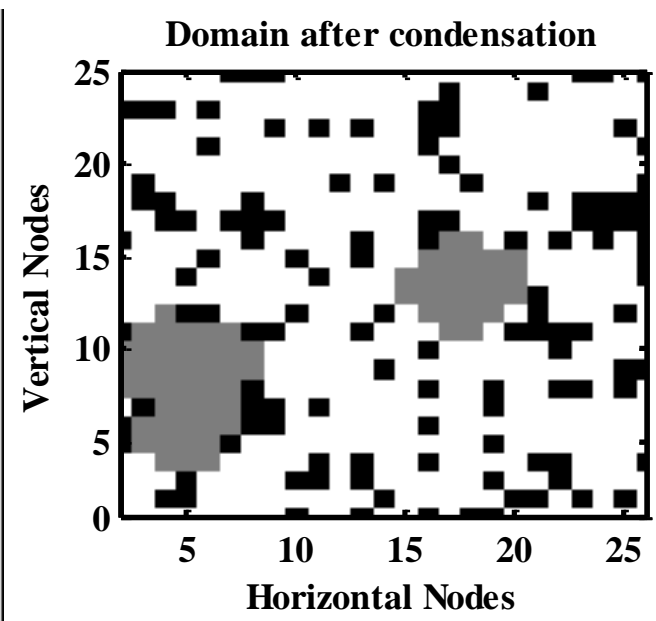

(b)

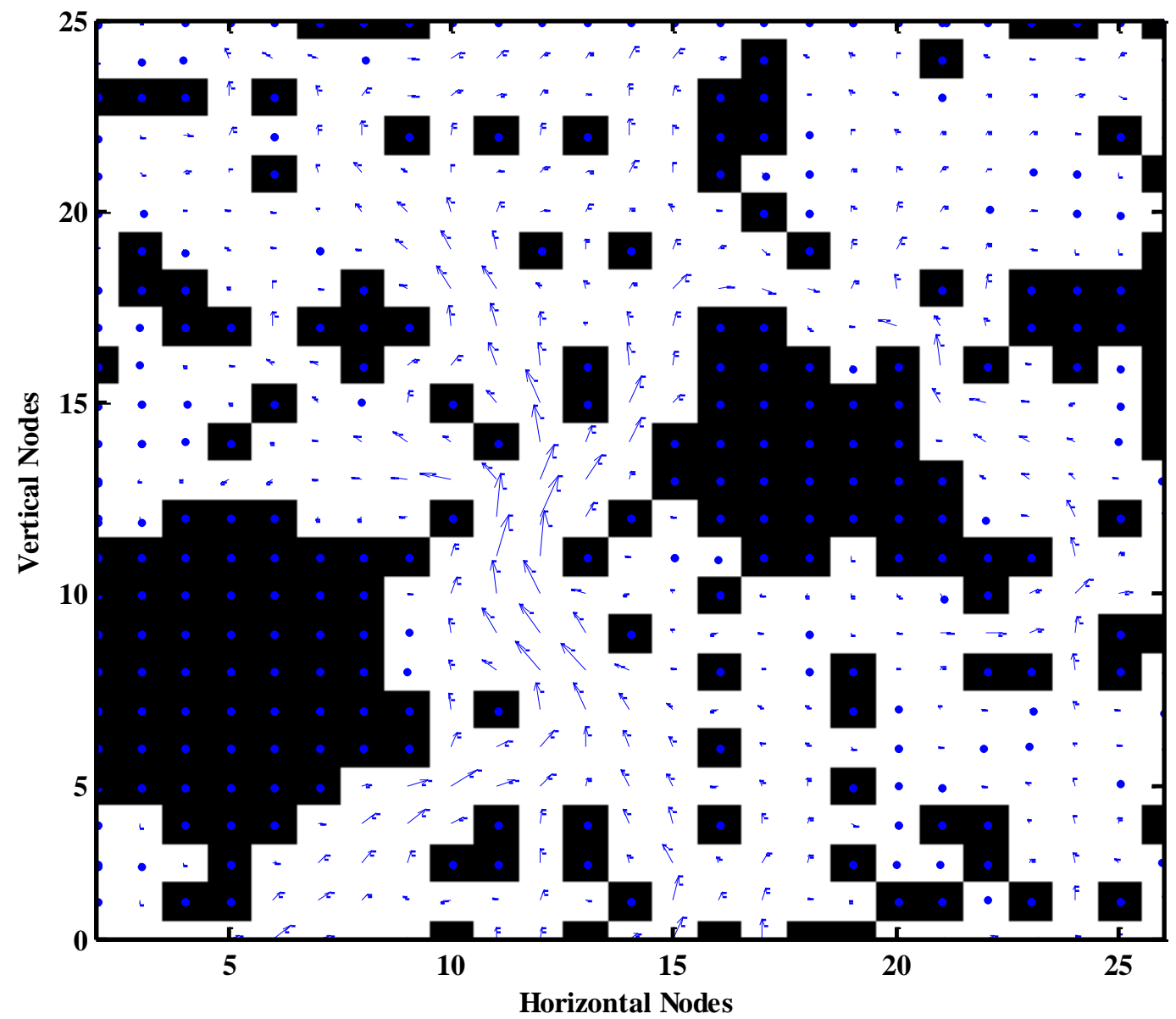

(c)

Figure 8 - Vapor phase mass transfer with a 10\% condensate (a) liquid formation (b) the voids occupied by liquid are shown in gray (c) Mass transfer in vapor phase, the effective tortuosity is 1.310 . 


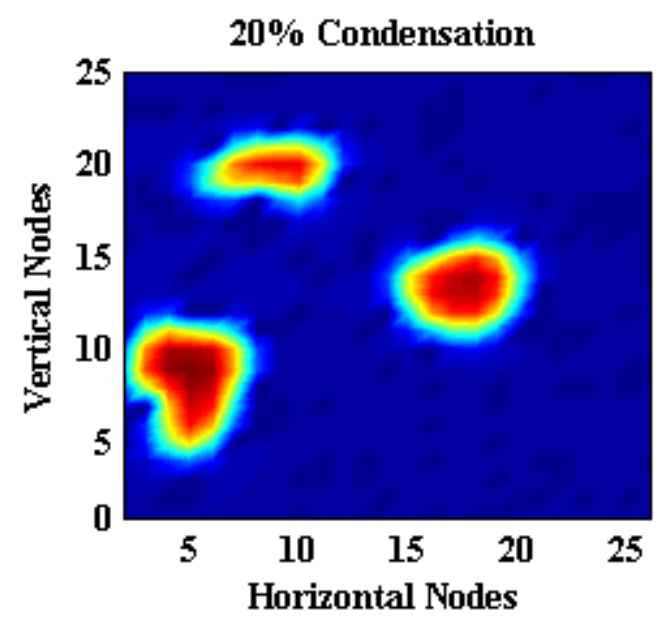

(a)

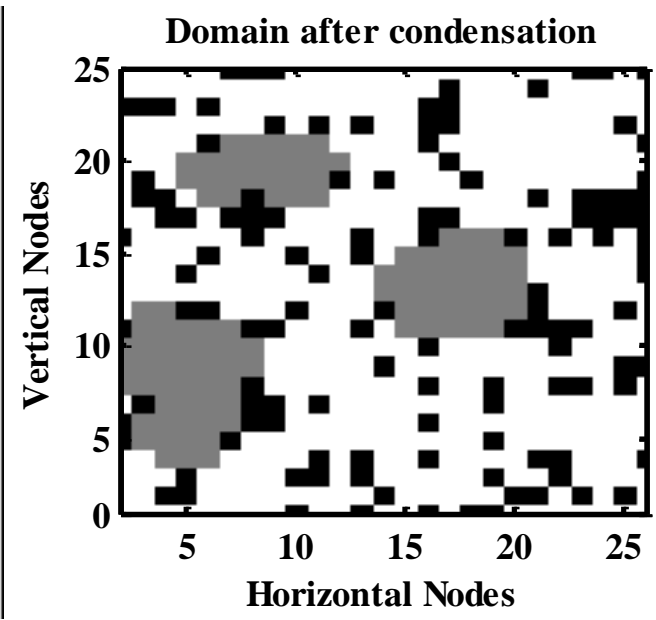

(b)

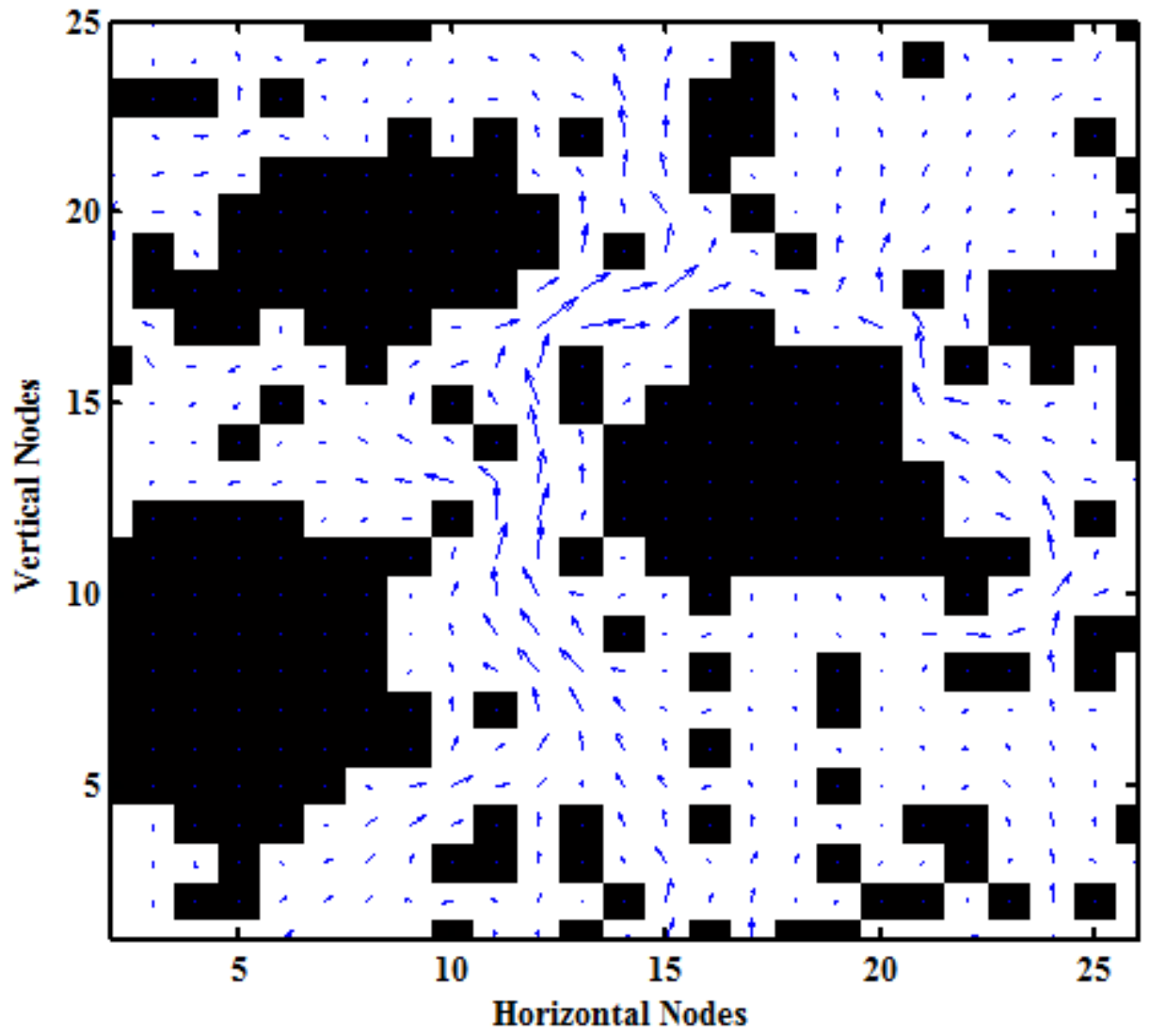

(c)

Figure 9 - Vapor phase mass transfer with a $20 \%$ condensate (a) liquid formation (b) condensate is shown in gray (c) Velocity field of the vapor phase, the effective tortuosity is 1.552. 


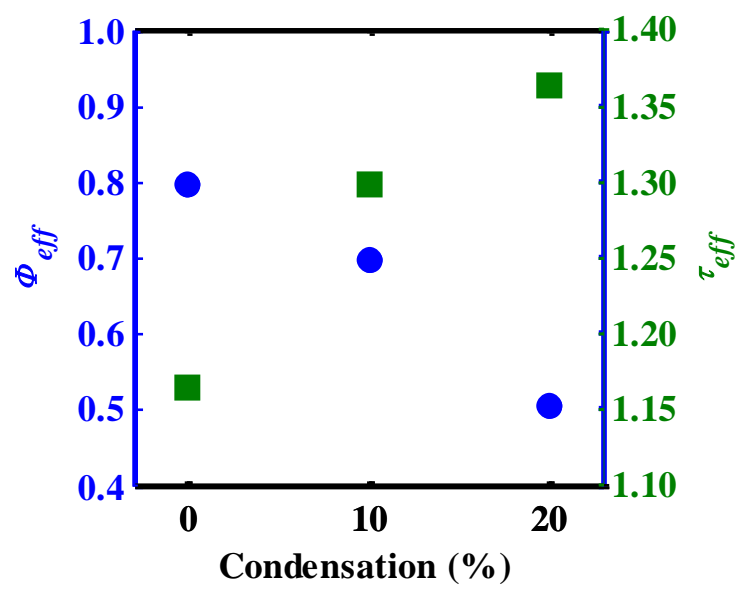

(a)

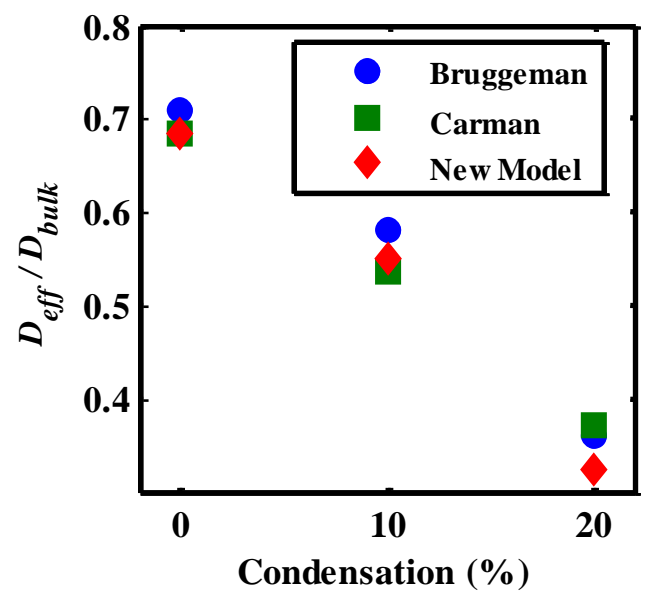

(b)

Figure 10 - Effects of liquid formation on mass transfer for a single example (a) effective porosity and tortuosity vs. condansate (b) diffusion ratio vs. condensate for several different multi-phase models. 


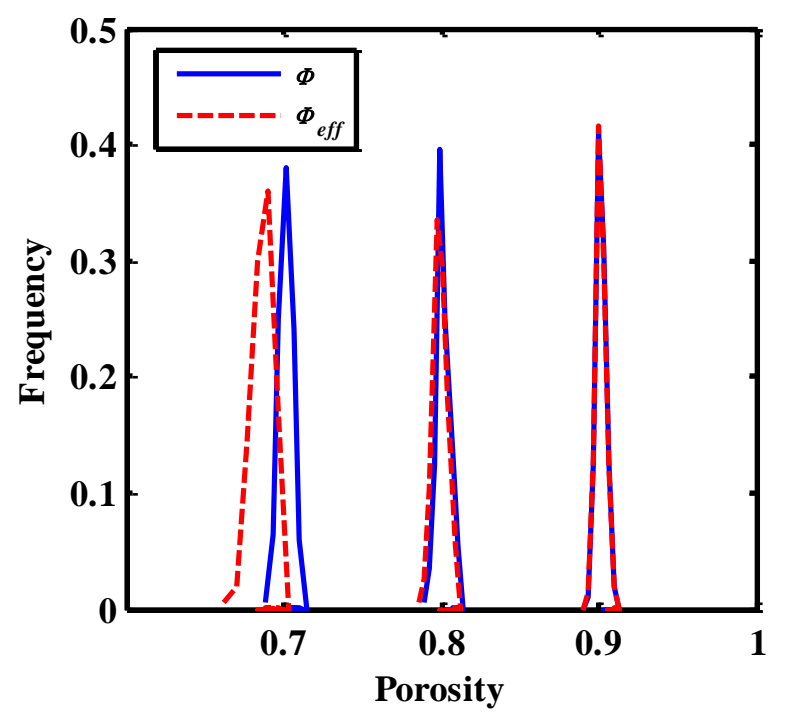

Figure 11 - Confidence level results for initially generated 100x100 porous media with $70 \%$, 80\%, and $90 \%$ porosity. 

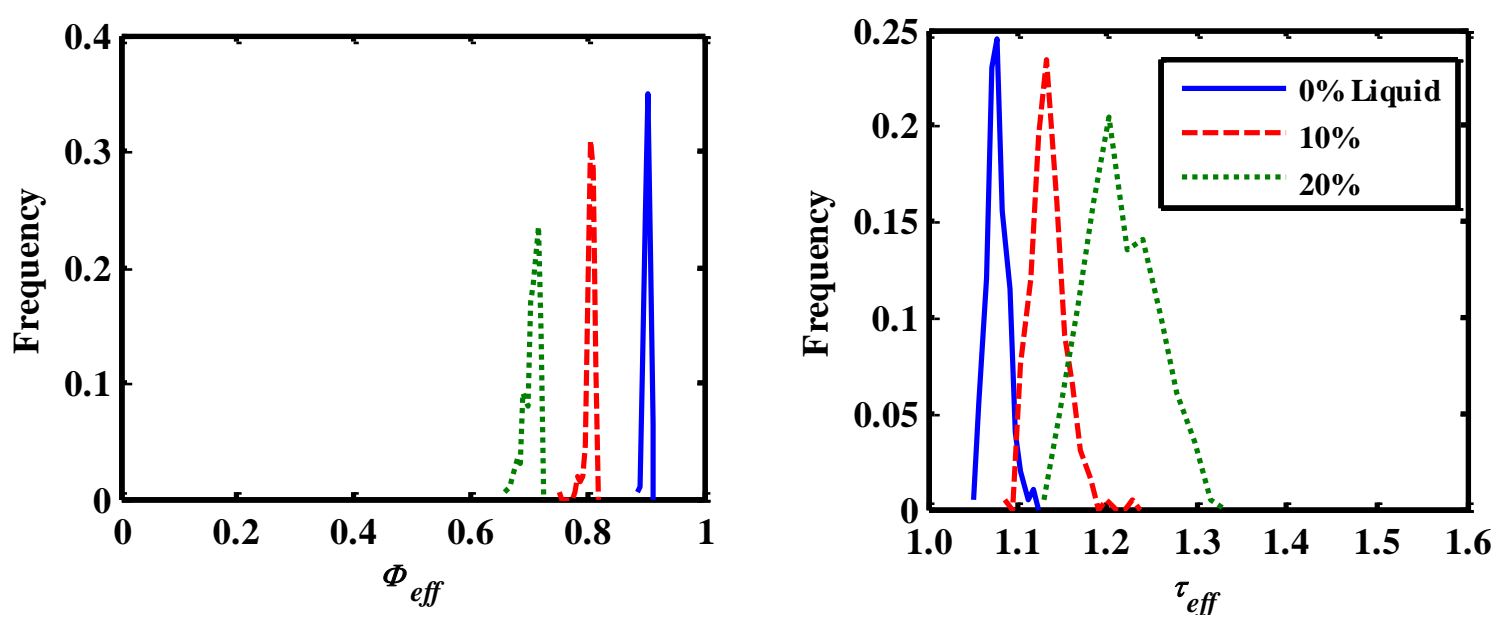

(a)
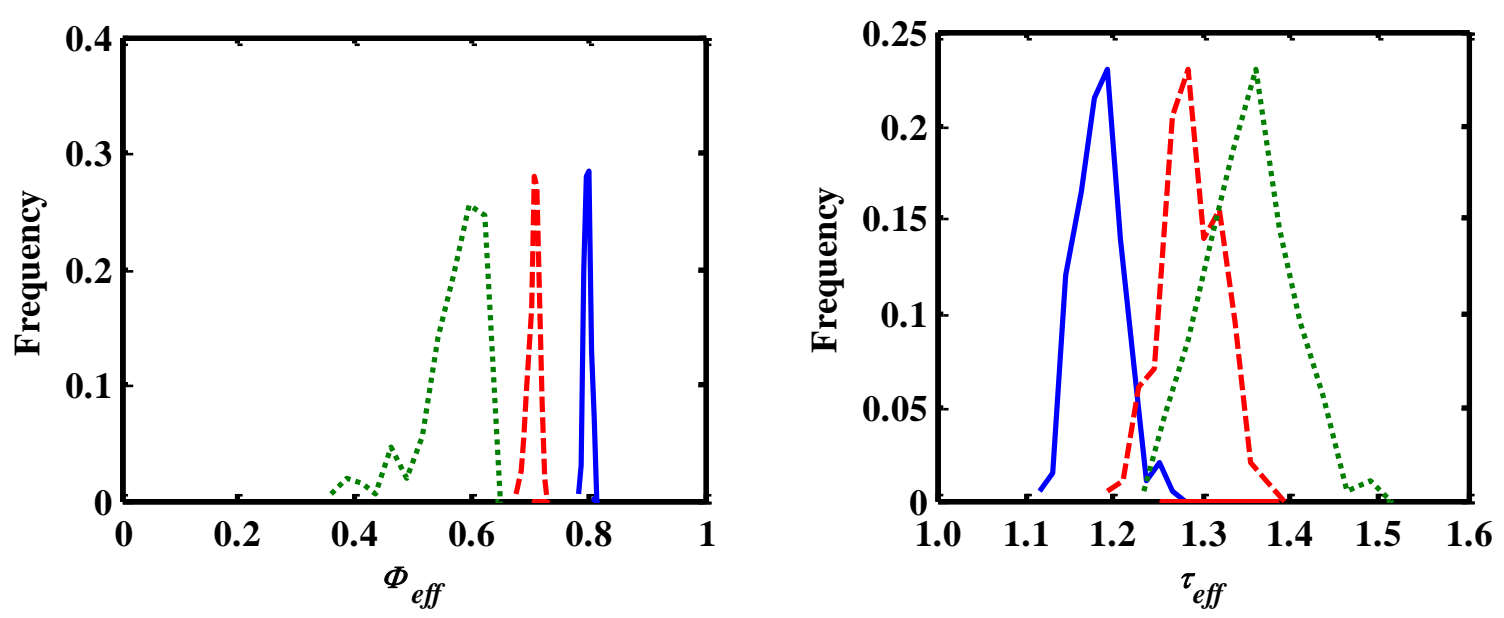

(b)
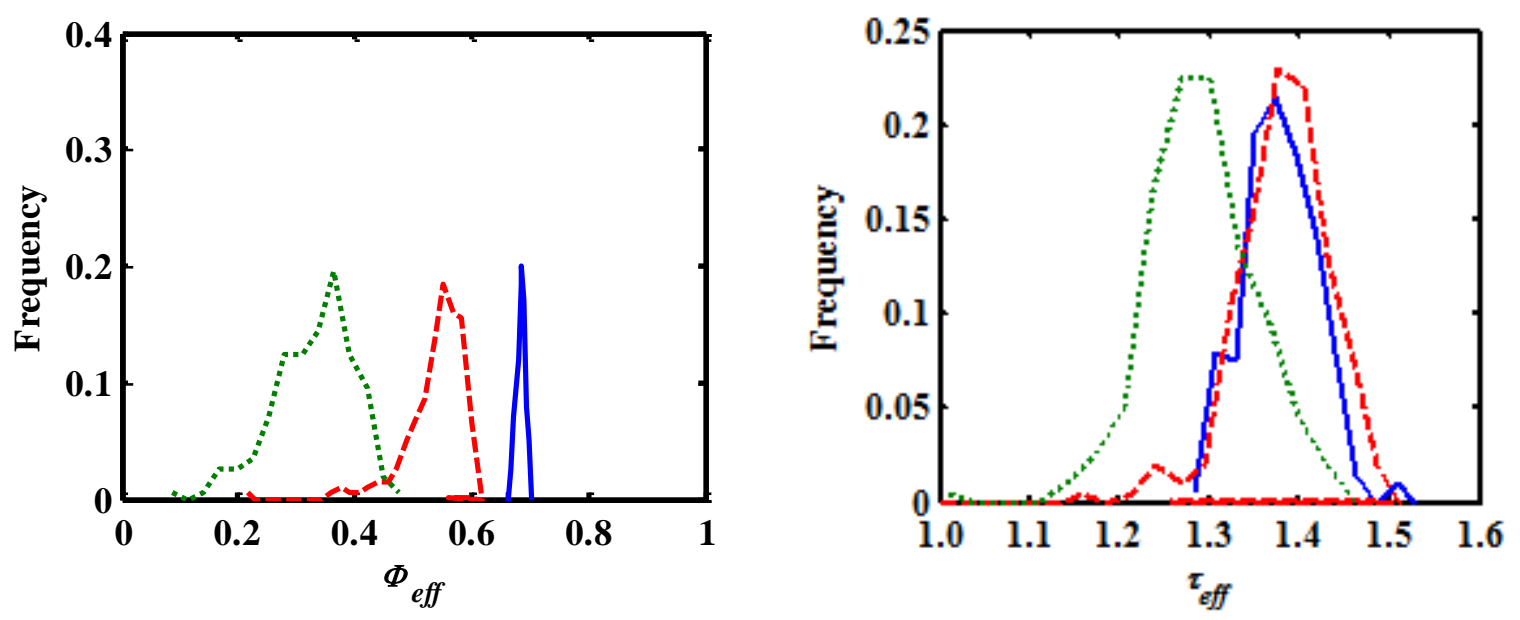

(c) 
Figure 12 - Confidence level results for SRAEs with (a) 90\%, (b) 80\%, and (c) $70 \%$ porosity. Figures on the left and right are probability distributions of effective porosity and tortuosity, respectively. 

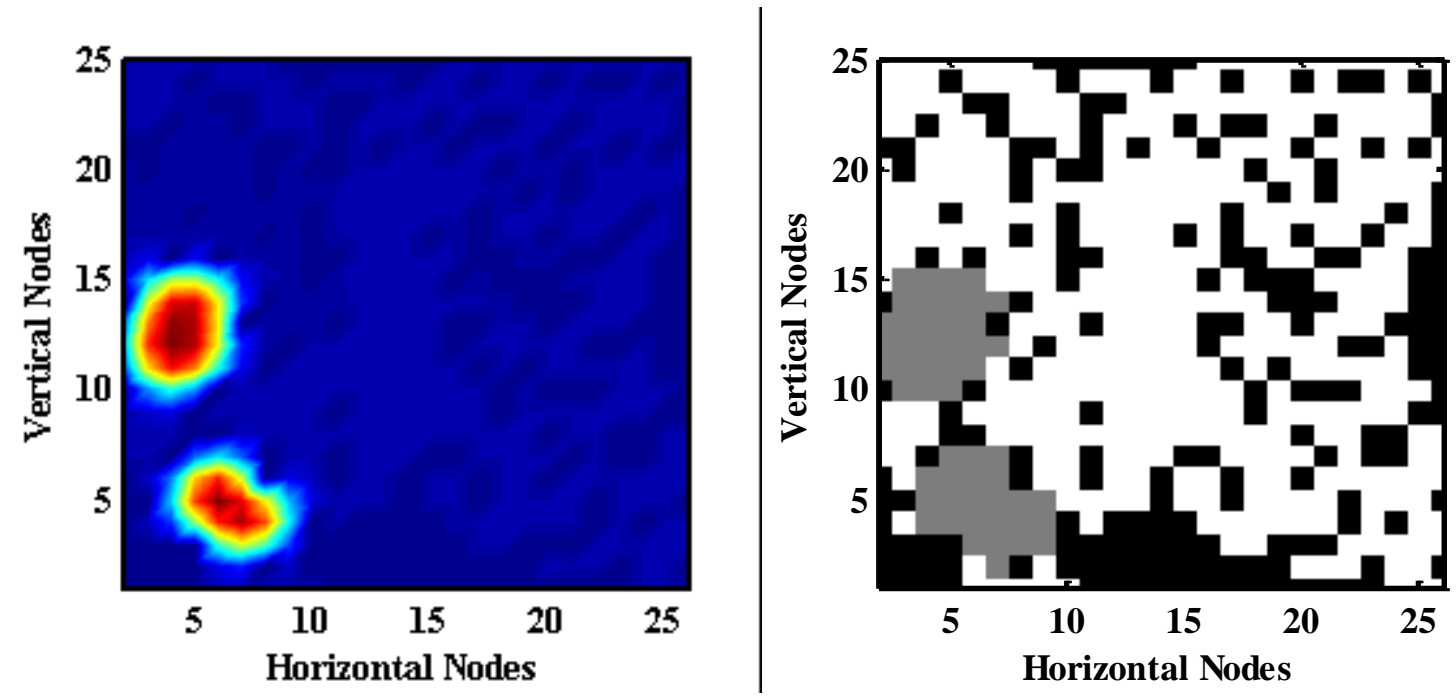

(a)
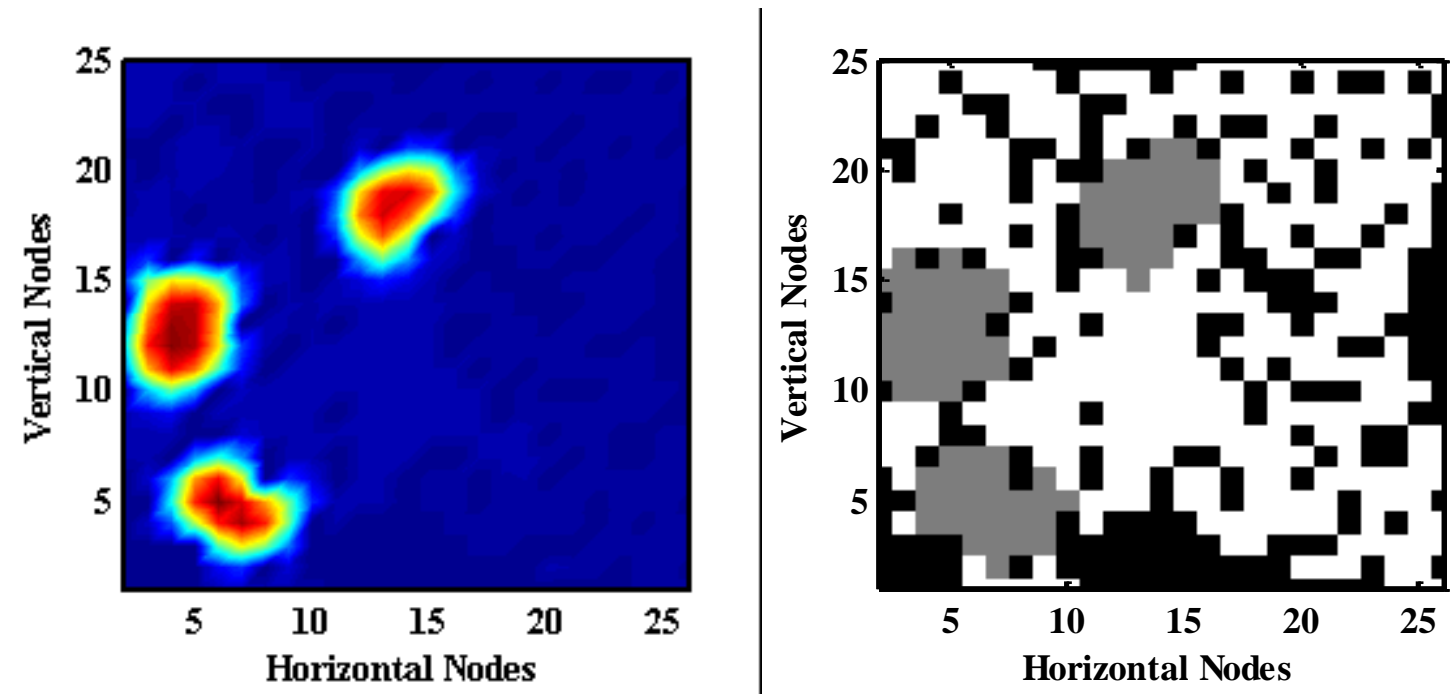

(b)

Figure 13 - Liquid formation in an example RAE for $70 \%$ target porosity (a) $10 \%$, (b) $20 \%$. 


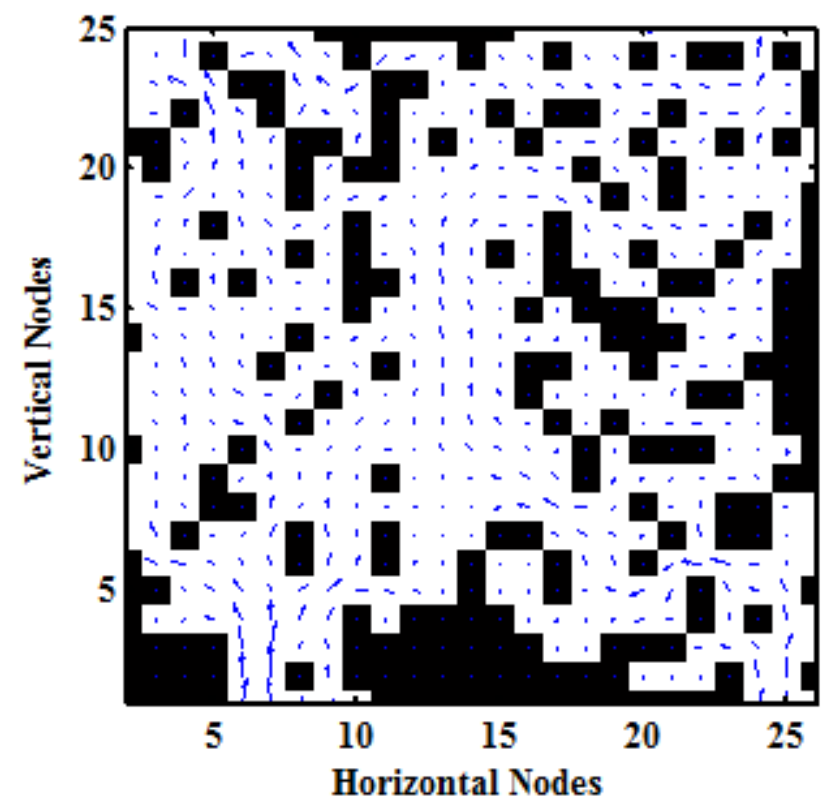

(a)

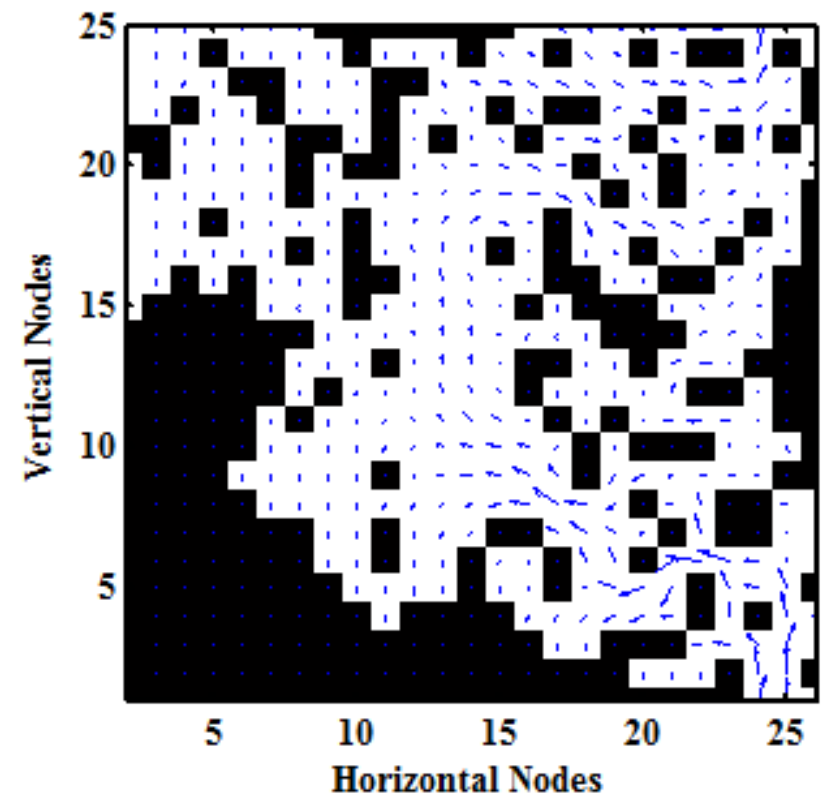

(b) 


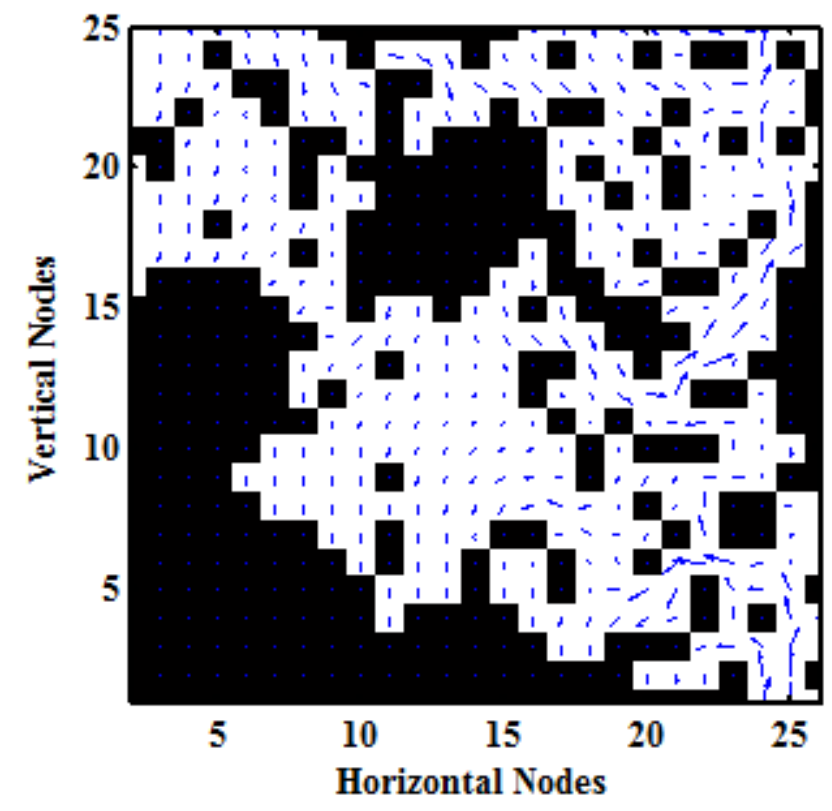

(c)

Figure 14 - Velocity fields in effective domains with (a) $0 \%$, (b) 10\%, (b) $20 \%$ condensate. Computed tortuosities are $1.403,1.448$, and 1.286, respectively. 


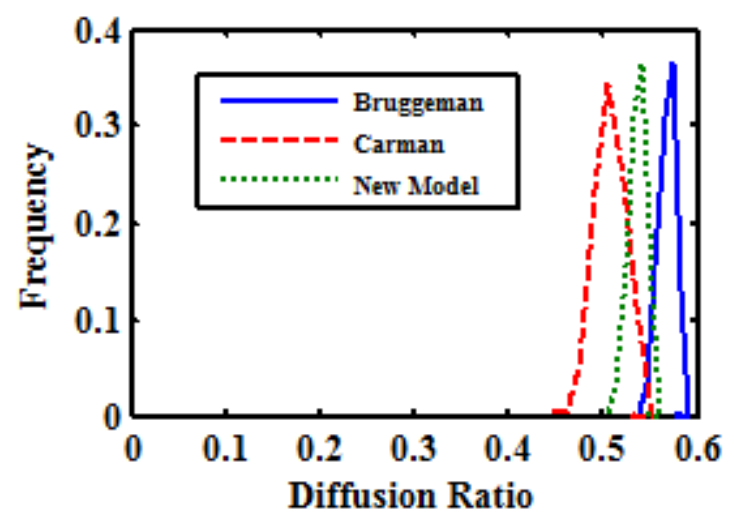

(a)

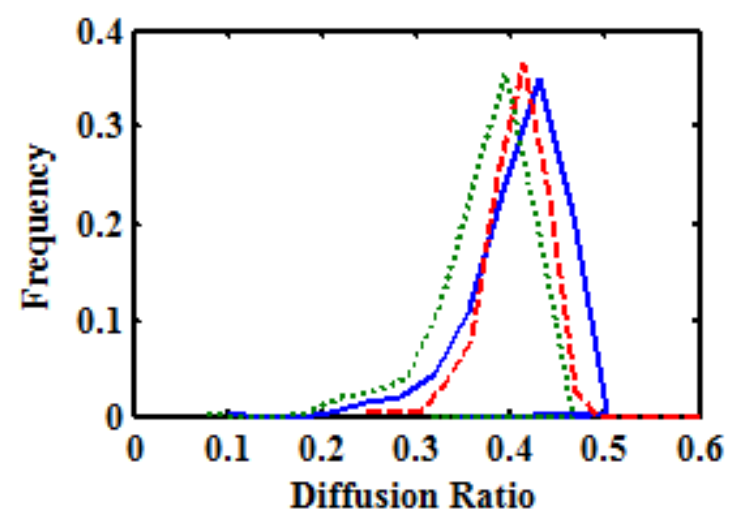

(b)

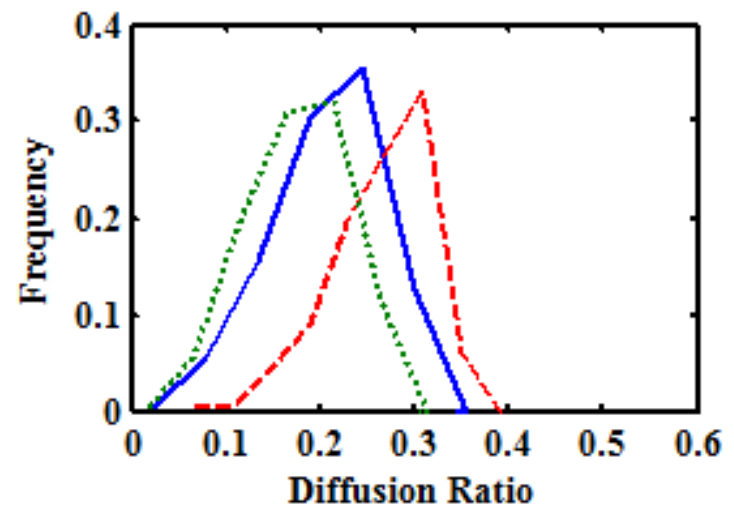

(c)

Figure 15 - Diffusion ratio probability distributions with a medium with (a) $0 \%$, (b) $10 \%$, (c) $20 \%$ liquid formation. 


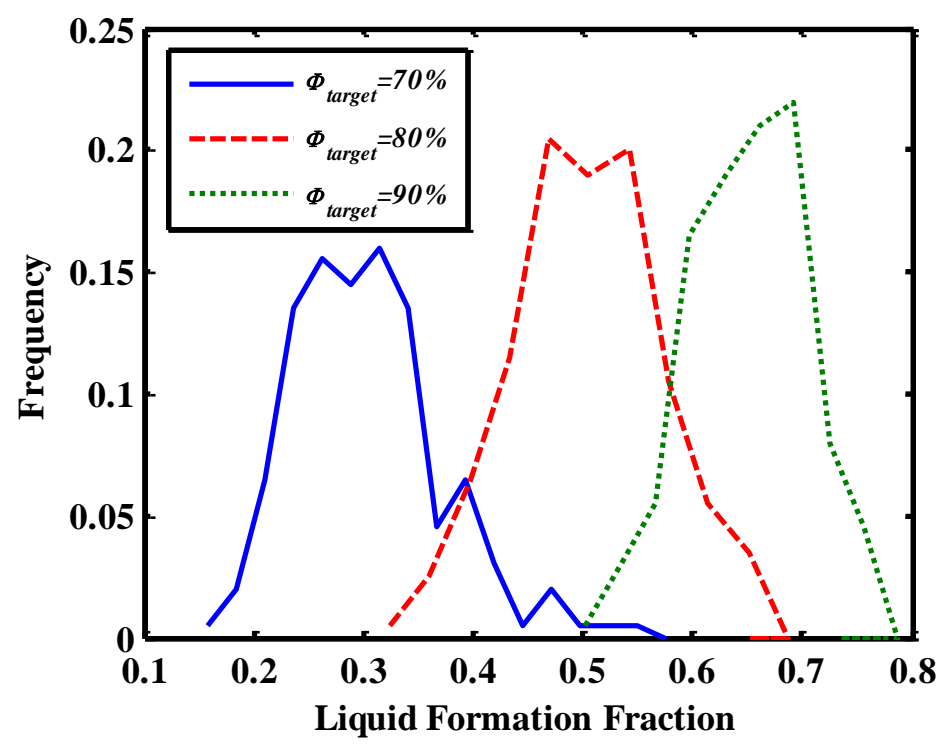

Figure 16 - Critical liquid formation probability distributions with a medium with $70 \%, 80 \%$, and $90 \%$ target porosity. 

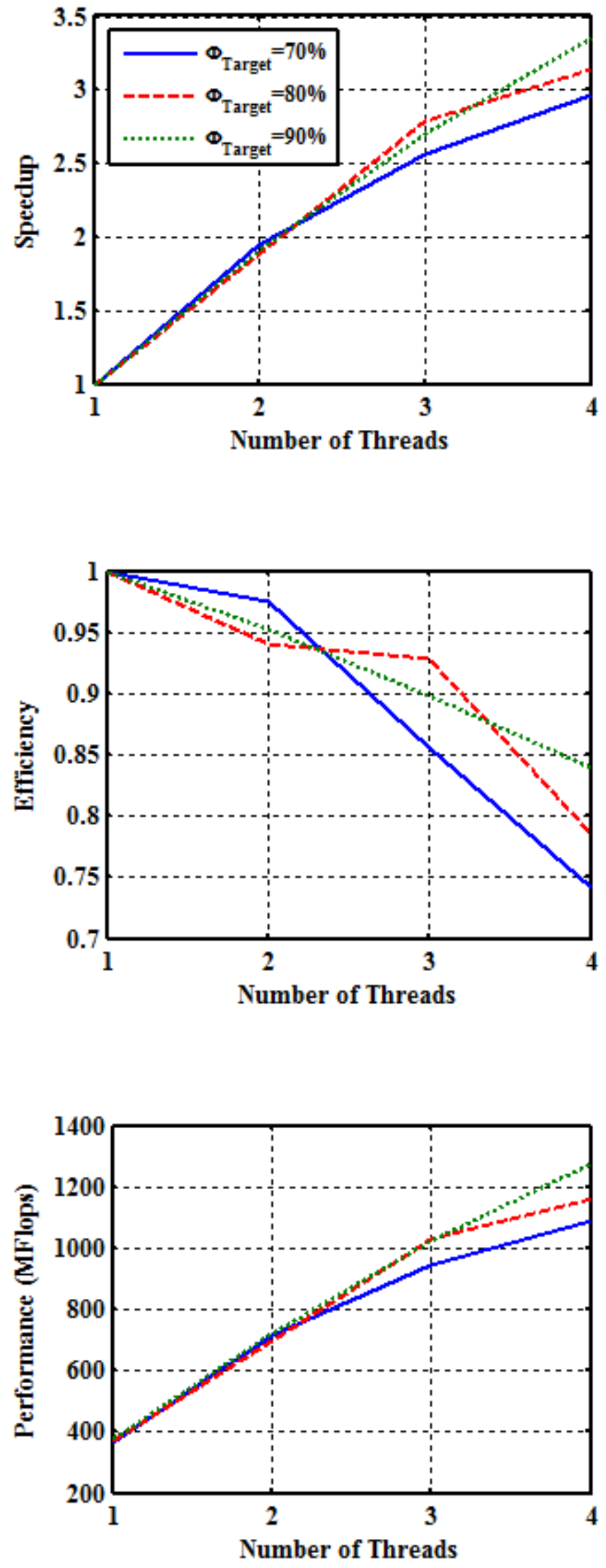
Figure 17 - Performance analysis for parallel application. 